\title{
Sulphur molecules in the circumstellar envelopes of M-type AGB stars ${ }^{\star}$
}

\author{
T. Danilovich, E. De Beck, J. H. Black, H. Olofsson, and K. Justtanont
}

\begin{abstract}
Onsala Space Observatory, Department of Earth and Space Sciences, Chalmers University of Technology, 43992 Onsala, Sweden e-mail: taissa@chalmers.se
\end{abstract}

Received 10 December 2015 / Accepted 25 January 2016

\begin{abstract}
Aims. The sulphur compounds $\mathrm{SO}$ and $\mathrm{SO}_{2}$ have not been widely studied in the circumstellar envelopes of asymptotic giant branch (AGB) stars. By presenting and modelling a large number of $\mathrm{SO}$ and $\mathrm{SO}_{2}$ lines in the low mass-loss rate M-type AGB star R Dor, and modelling the available lines of those molecules in a further four M-type AGB stars, we aim to determine their circumstellar abundances and distributions.

Methods. We use a detailed radiative transfer analysis based on the accelerated lambda iteration method to model circumstellar SO and $\mathrm{SO}_{2}$ line emission. We use molecular data files for both $\mathrm{SO}$ and $\mathrm{SO}_{2}$ that are more extensive than those previously available. Results. Using $17 \mathrm{SO}$ lines and $98 \mathrm{SO}_{2}$ lines to constrain our models for R Dor, we find an SO abundance of $(6.7 \pm 0.9) \times 10^{-6}$ and an $\mathrm{SO}_{2}$ abundance of $5 \times 10^{-6}$ with both species having high abundances close to the star. We also modelled ${ }^{34} \mathrm{SO}$ and found an abundance of $(3.1 \pm 0.8) \times 10^{-7}$, giving an ${ }^{32} \mathrm{SO} /{ }^{34} \mathrm{SO}$ ratio of $21.6 \pm 8.5$. We derive similar results for the circumstellar $\mathrm{SO}$ and $\mathrm{SO}_{2}$ abundances and their distributions for the low mass-loss rate object W Hya. For the higher mass-loss rate stars, we find shell-like SO distributions with peak abundances that decrease and peak abundance radii that increase with increasing mass-loss rate. The positions of the peak SO abundance agree very well with the photodissociation radii of $\mathrm{H}_{2} \mathrm{O}$. We also modelled $\mathrm{SO}_{2}$ in two higher mass-loss rate stars but our models for these were less conclusive.

Conclusions. We conclude that for the low mass-loss rate stars, the circumstellar $\mathrm{SO}$ and $\mathrm{SO}_{2}$ abundances are much higher than predicted by chemical models of the extended stellar atmosphere. These two species may also account for all the available sulphur. For the higher mass-loss rate stars we find evidence that SO is most efficiently formed in the circumstellar envelope, most likely through the photodissociation of $\mathrm{H}_{2} \mathrm{O}$ and the subsequent reaction between $\mathrm{S}$ and $\mathrm{OH}$. The S-bearing parent molecule does not appear to be $\mathrm{H}_{2} \mathrm{~S}$. The $\mathrm{SO}_{2}$ models for the higher mass-loss rate stars are less conclusive, but suggest an origin close to the star for this species. This is not consistent with current chemical models. The combined circumstellar $\mathrm{SO}$ and $\mathrm{SO}_{2}$ abundances are significantly lower than that of sulphur for these higher mass-loss rate objects.
\end{abstract}

Key words. stars: mass-loss - stars: AGB and post-AGB - circumstellar matter - stars: evolution

\section{Introduction}

Low- to intermediate-mass stars eventually evolve from the main sequence to the asymptotic giant branch (AGB). AGB stars lose mass rapidly, producing a circumstellar envelope (CSE) of atomic and molecular matter and dust, rich in chemical diversity. The variety and abundances of molecules that can be found in the CSEs of AGB stars depend on the chemistry of the individual star. For example, carbon stars, which have carbon-to-oxygen ratio $\mathrm{C} / \mathrm{O}>1$, are most likely to have a variety of $\mathrm{C}$-bearing molecules in their CSEs (e.g. Gong et al. 2015), while oxygenrich M-type stars, with $\mathrm{C} / \mathrm{O}<1$, are more likely to contain a variety of O-bearing molecules (e.g. Justtanont et al. 2012).

$\mathrm{SO}$ and $\mathrm{SO}_{2}$ are two such O-bearing molecules. They are thought to exist in shells in the CSE, having been formed through the photodissociation of the parent molecule $\mathrm{H}_{2} \mathrm{~S}$ and subsequent reactions with $\mathrm{O}$ and $\mathrm{OH}$ (Cherchneff 2006; Willacy \& Millar 1997). Observations of the red supergiant VY CMa by Adande et al. (2013) contradict this view, however; the

\footnotetext{
* Herschel is an ESA space observatory with science instruments provided by European-led Principal Investigator consortia and with important participation from NASA.
}

modelling results show small, concentrated envelopes of SO and $\mathrm{SO}_{2}$ and indications that $\mathrm{SO}_{2}$ may itself be formed directly, rather than being a photodissociation product of $\mathrm{H}_{2} \mathrm{~S}$ or another molecule and are found in a hollow shell around the star. Similarly, when Decin et al. (2010a) modelled $\mathrm{SO}_{2}$ emission around the AGB star IK Tau, they had difficulty reconciling their observations with a shell model.

The Yamamura et al. (1999) ISO/SWS detections of the $7.4 \mu \mathrm{m} v_{3} \mathrm{SO}_{2}$ band in a few AGB stars suggest that $\mathrm{SO}_{2}$ is formed in the warmest regions of the CSE. Analysis of these data by Yamamura et al. (1999) and Cami et al. (1999) indicates that the $\mathrm{SO}_{2}$ is mostly likely formed within a few stellar radii of the star at a temperature of $\sim 600 \mathrm{~K}$. Cami et al. (1999) also find that the excitation of $\mathrm{SO}_{2}$ to the $v_{3}$ band varies with pulsation period. Their simple models put the outer radius of $\mathrm{SO}_{2}$ within $\sim 5 R_{*}$.

In this paper we present new observations of circumstellar $\mathrm{SO}$ and $\mathrm{SO}_{2}$ from an APEX spectral survey of the M-type AGB star R Dor. We combine these results with $\mathrm{SO}$ and $\mathrm{SO}_{2}$ detections from Herschel/HIFI, developing comprehensive models of the $\mathrm{SO}$ and $\mathrm{SO}_{2}$ distributions around $\mathrm{R}$ Dor using $17 \mathrm{SO}$ lines and $98 \mathrm{SO}_{2}$ lines, all spectrally resolved. 
Table 1. Basic information about our five sources.

\begin{tabular}{ccccc}
\hline \hline Star & RA & Dec & Variability & Spec type \\
\hline IK Tau & 035328.87 & +112421.7 & M & M9 \\
R Dor & 043645.59 & -620437.8 & SRB & M8e \\
TX Cam & 050050.39 & +561052.6 & M & M8.5 \\
W Hya & 134902.00 & -282203.5 & M & M7.5-9e \\
R Cas & 235824.87 & +512319.7 & M & M6.5-9e \\
\hline
\end{tabular}

Notes. RA and Dec are given in J2000 co-ordinates. The variability types are $\mathrm{M}=$ Mira variable, $\mathrm{SRB}=$ semi-regular variable type $\mathrm{B}$.

We also model the sparse detections of $\mathrm{SO}$ and $\mathrm{SO}_{2}$ emission towards the other M-type AGB stars observed with Herschel/HIFI, supplemented with archival data where available.

\section{Sample and observations}

The stars included in this study come from the sample of Mtype AGB stars observed as part of the HIFISTARS guaranteed time key programme (Justtanont et al. 2012, and see Sect. 2.2 for details). The $\mathrm{OH} / \mathrm{IR}$ stars are excluded, as is Mira, which has a complicated and asymmetric CSE induced by a white dwarf companion (see Ramstedt et al. 2014). That leaves a sample of five M-stars, four of which had $\mathrm{SO}$ and $\mathrm{SO}_{2}$ lines detected by HIFI. The remaining star, TX Cam, has previously been detected in $\mathrm{SO}$ at lower frequencies.

Some basic information about the five sources is given in Table 1.

\subsection{APEX data}

We performed a spectral survey of $\mathrm{R}$ Dor in the ranges 213-321.5 GHz and 338.5-368.5 GHz $(\lambda=0.8-1.4 \mathrm{~mm})$ using the Swedish Heterodyne Facility Instrument (SHeFI; Vassilev et al. 2008) on the Atacama Pathfinder Experiment telescope (APEX). The data were observed over several observing seasons between May 2011 and June 2015. The observations were carried out using beam switching with a standard beam throw of $3^{\prime}$. A detailed description of this survey will be presented by De Beck et al. (in prep.).

Data reduction was carried out using the GILDAS/CLAss ${ }^{1}$ package. Scans with very unstable baselines were ignored and bad channels were blanked. After masking the regions with line emission, polynomial baselines of typically first degree were subtracted from the averaged spectra to obtain a $0 \mathrm{~K}$ baseline. Rms noise levels throughout the survey are around $2-10 \mathrm{mK}$ at a velocity resolution of $1 \mathrm{~km} \mathrm{~s}^{-1}$. The spectra were then converted to main beam temperatures using efficiency correction factors of $\eta_{\mathrm{mb}}=0.75$ for $v<270 \mathrm{GHz}, \eta_{\mathrm{mb}}=0.74$ for $270<v<320 \mathrm{GHz}$, and $\eta_{\mathrm{mb}}=0.73$ for $v>320 \mathrm{GHz}$. The halfpower beam-widths were calculated using the general formula

$$
\theta=7.8\left(\frac{800}{v}\right)
$$

where $v$ is in $\mathrm{GHz}$ and $\theta$ is in arcseconds. The beam-widths across our frequency range are between 17-29".

The detections of SO using APEX are listed in Table 2 and the $\mathrm{SO}_{2}$ detections are listed in Table C.1. There were also some

\footnotetext{
1 http://wWw.iram.fr/IRAMFR/GILDAS/
}

Table 2. SO observations towards R Dor using APEX, listed in order of descending energy of the upper level.

\begin{tabular}{crrrc}
\hline \hline Transition & $\begin{array}{r}v \\
{[\mathrm{GHz}]}\end{array}$ & $\begin{array}{r}E_{\mathrm{up}} \\
{[\mathrm{K}]}\end{array}$ & $\begin{array}{c}\theta \\
{\left[{ }^{\prime \prime}\right]}\end{array}$ & $\begin{array}{c}I_{\mathrm{mb}} \\
{\left[\mathrm{K} \mathrm{km} \mathrm{s}^{-1}\right]}\end{array}$ \\
\hline $8_{8} \rightarrow 7_{7}$ & 344.311 & 88 & 18 & 5.04 \\
$8_{7} \rightarrow 7_{6}$ & 340.714 & 81 & 18 & 4.59 \\
$8_{9} \rightarrow 7_{8}$ & +346.528 & 79 & 18 & 4.54 \\
$7_{7} \rightarrow 6_{6}$ & 301.286 & 71 & 21 & 4.74 \\
$7_{6} \rightarrow 6_{5}$ & 296.550 & 65 & 21 & 4.12 \\
$7_{8} \rightarrow 6_{7}$ & 304.078 & 62 & 21 & 6.40 \\
$6_{6} \rightarrow 5_{5}$ & 258.256 & 57 & 24 & 3.49 \\
$6_{5} \rightarrow 5_{4}$ & 251.826 & 51 & 25 & 3.13 \\
$6_{7} \rightarrow 5_{6}$ & 261.844 & 48 & 24 & 5.20 \\
$5_{5} \rightarrow 4_{4}$ & 215.221 & 44 & 29 & 2.25 \\
$5_{6} \rightarrow 4_{5}$ & 219.949 & 35 & 28 & 4.21 \\
$3_{3} \rightarrow 2_{3}$ & 339.341 & 26 & 18 & 0.125 \\
$2_{2} \rightarrow 1_{2}$ & 309.502 & 19 & 20 & 0.100 \\
\hline
\end{tabular}

Notes. ${ }^{(\dagger)}$ Indicates a line overlap with $\mathrm{SO}_{2}$.

Table 3. $\mathrm{SO}$ and $\mathrm{SO}_{2}$ isotopologue observations towards $\mathrm{R}$ Dor using APEX.

\begin{tabular}{lcrrrc}
\hline \hline & Transition & $\begin{array}{r}v \\
{[\mathrm{GHz}]}\end{array}$ & $\begin{array}{r}E_{\text {up }} \\
{[\mathrm{K}]}\end{array}$ & $\begin{array}{r}\theta \\
{\left[{ }^{\prime \prime}\right]}\end{array}$ & $\begin{array}{c}I_{\mathrm{mb}} \\
{\left[\mathrm{K} \mathrm{km} \mathrm{s}^{-1}\right]}\end{array}$ \\
\hline${ }^{34} \mathrm{SO}$ & $8_{9} \rightarrow 7_{8}$ & 339.857 & 77.3 & 18 & 0.26 \\
& $7_{7} \rightarrow 6_{6}$ & 295.396 & 69.9 & 21 & 0.20 \\
& $7_{8} \rightarrow 6_{7}$ & 298.258 & 61.1 & 21 & 0.42 \\
& $6_{6} \rightarrow 5_{5}$ & 253.207 & 55.7 & 25 & 0.26 \\
& $6_{7} \rightarrow 5_{6}$ & 256.878 & 46.7 & 24 & 0.38 \\
& $5_{6} \rightarrow 4_{5}$ & 215.840 & 34.4 & 29 & 0.18 \\
${ }^{34} \mathrm{SO}_{2}$ & $20_{0,20} \rightarrow 19_{1,19}$ & 357.102 & 184.6 & 17 & 0.25 \\
& $17_{3,15} \rightarrow 17_{2,16}$ & 279.075 & 161.9 & 22 & 0.11 \\
$\mathrm{SO}^{18} \mathrm{O}$ & $6_{3,3} \rightarrow 5_{2,4}$ & 362.158 & 40.6 & 17 & 0.24 \\
& $35_{10,26} \rightarrow 36_{9,27}$ & 288.482 & 786.3 & 22 & 0.19 \\
& $19_{3,17} \rightarrow 19_{2,18}$ & 288.270 & 186.8 & 22 & 0.24 \\
& $18_{0,18} \rightarrow 17_{1,17}$ & 303.476 & 143.4 & 21 & 0.18 \\
& $17_{2,16} \rightarrow 16_{2,15}$ & 303.155 & 141.3 & 21 & 0.28 \\
& $14_{4,10} \rightarrow 14_{3,11}$ & 344.874 & 129.6 & 18 & 0.24 \\
\hline
\end{tabular}

detections of $\mathrm{SO}$ and $\mathrm{SO}_{2}$ isotopologues: ${ }^{34} \mathrm{SO}$, and tentative detections of ${ }^{34} \mathrm{SO}_{2}$ and $\mathrm{SO}^{18} \mathrm{O}$. We model ${ }^{34} \mathrm{SO}$, but are unable to perform a full radiative transfer analysis for the other isotopologues. See Table 3 for a list of isotopologue detections and for the full discussion, see Sect. 3.2.4.

In terms of other S-bearing molecules, there were no conclusive detections of either CS (out of three possible transitions in the range $J_{\text {up }}=5$ to $J_{\text {up }}=7$ ) or SiS (out of nine possible transitions in the range $J_{\text {up }}=12$ to $\left.J_{\text {up }}=20\right)$. There was a tentative detection of $\mathrm{CS}(6 \rightarrow 5)$ but it is blended with ${ }^{29} \mathrm{SiO}(7 \rightarrow 6, v=3)$ line and hence allows no reliable conclusion on the detection of $\mathrm{CS}$. (We note that there are several other detections of ${ }^{29} \mathrm{SiO}$ in the survey, but none of CS.) No other S-bearing molecules were detected in this survey.

\subsection{HIFI data}

R Dor, IK Tau, R Cas, TX Cam and W Hya were observed as part of the HIFISTARS guaranteed time key programme, using the Herschel/HIFI instrument (de Graauw et al. 2010) to observe emission lines with high spectral resolution. The full results are presented in detail in Justtanont et al. (2012). Since those data were published, there have been updates to the main beam 
Table 4. Stellar properties and input from CO models.

\begin{tabular}{lccccc}
\hline \hline & IK Tau & R Dor & TX Cam & W Hya & R Cas \\
\hline$L_{*}\left[\mathrm{~L}_{\odot}\right]$ & 7700 & 6500 & 8600 & 5400 & 8700 \\
$D[\mathrm{pc}]$ & 265 & 59 & 380 & 78 & 176 \\
$v_{\mathrm{LSR}}\left[\mathrm{km} \mathrm{s}^{-1}\right]$ & 34 & 7 & 11.4 & 40.5 & 25 \\
$T_{*}[\mathrm{~K}]$ & 2100 & 2400 & 2400 & 2500 & 3000 \\
$R_{\text {in }}\left[10^{14} \mathrm{~cm}\right]$ & 2.0 & 1.9 & 2.2 & 2.0 & 2.2 \\
$\tau_{10}$ & 1.0 & 0.03 & 0.4 & 0.07 & 0.09 \\
$\dot{M}\left[10^{-7} M_{\odot} \mathrm{yr}^{-1}\right]$ & 50 & 1.6 & 40 & 1 & 8 \\
$v_{\infty}\left[\mathrm{km} \mathrm{s}^{-1}\right]$ & 17.5 & 5.7 & 17.5 & 7.5 & 10.5 \\
$\beta$ & 1.5 & 1.5 & 2.0 & 5.0 & 2.5 \\
\hline
\end{tabular}

Notes. $\tau_{10}$ is the dust optical depth at $10 \mu \mathrm{m}$.

efficiencies (Mueller et al. 2014 ${ }^{2}$ ) and for this work we have rereduced the HIFI data to take this into account (using $\mathrm{HIPE}^{3}$ version 12.1, Ott 2010). We have also identified three additional $\mathrm{SO}_{2}$ lines that were not included in Justtanont et al. (2012). The detected $\mathrm{SO}$ and $\mathrm{SO}_{2}$ HIFI lines are listed in Table C.2. We note that no SO or $\mathrm{SO}_{2}$ lines were detected with HIFI in TX Cam.

\subsection{Archival data}

To supplement the HIFI data for IK Tau, R Cas, W Hya, and TX Cam, we have used observations found in the literature. These are listed in Table C.3. As the older data generally covers lower-energy transitions than those observed by HIFI, we are better able to constrain our models over a larger energy range. This is particularly important for R Cas, IK Tau and TX Cam where the HIFI lines (or non-detections in the case of TX Cam) are clustered close together energetically.

\section{Modelling}

\subsection{Modelling procedure}

We perform detailed radiative transfer modelling of the molecular emission lines using an accelerated lambda iteration method code (ALI), which has been previously described and implemented by e.g. Maercker et al. (2008), Schöier et al. (2011), Danilovich et al. (2014). ALI is particularly useful in this work as it is able to take into account extensive descriptions of molecular properties - such as large numbers of energy levels and transitions - while still fully solving the statistical equilibrium equations and taking temperature and velocity profiles into account.

We assume a smoothly expanding spherical CSE produced by a constant mass-loss rate. The molecules are located in this CSE until they eventually become photodissociated. They are excited by collisions with $\mathrm{H}_{2}$ molecules and through radiation from the star, the dust, and the cosmic microwave background. ALI input parameters such as the kinetic temperature distribution, dust temperature, and dust optical depth, are taken from CO modelling and, where applicable, are listed in Table 4. For R Dor, R Cas, IK Tau, and TX Cam Maercker et al. (in prep.) performed detailed radiative transfer modelling of the $\mathrm{CO}$ and $\mathrm{H}_{2} \mathrm{O}$ lines and we use their results in our modelling. We based our CO model of W Hya on the results of Khouri et al. (2014a),

\footnotetext{
2 http://herschel.esac.esa.int/twiki/pub/Public/ HifiCalibrationWeb/HifiBeamReleaseNote_Sep2014.pdf

3 http://www. cosmos.esa.int/web/herschel/

data-processing-overview
}

but generated a CO model using the same code as in Maercker et al. (in prep.) for consistency between the stars.

We calculated the best fit model for each star and molecule using a $\chi^{2}$ statistic, which we define as

$\chi^{2}=\sum_{i=1}^{N} \frac{\left(I_{\mathrm{mod}, i}-I_{\mathrm{obs}, i}\right)^{2}}{\sigma_{i}^{2}}$

where $I$ is the integrated line intensity, $\sigma$ is the uncertainty in the observations, and $N$ is the number of lines being modelled. We also calculate a reduced $\chi^{2}$ value such that $\chi_{\text {red }}^{2}=\chi^{2} /(N-p)$ where $p$ is the number of free parameters.

After testing both centrally-peaked and shell-like abundance distributions, we came to the conclusion that the best radial abundance distribution profiles for both $\mathrm{SO}$ and $\mathrm{SO}_{2}$ in $\mathrm{R}$ Dor and W Hya were Gaussian profiles of the form

$f=f_{\mathrm{p}} \exp \left(-\left(\frac{r}{R_{e}}\right)^{2}\right)$

where $f_{\mathrm{p}}$ is the peak abundance at the inner radius, and $R_{e}$ is the $e$-folding radius, the radius at which the abundance has dropped by a factor of $1 / e$.

In the cases of IK Tau and R Cas, we found that a shell model was a better fit to the observed SO lines. As such, we modelled IK Tau and R Cas assuming a Gaussian shell for the abundance distribution of the form

$f=f_{\mathrm{p}} \exp \left(-4 \frac{\left(r-R_{\mathrm{p}}\right)^{2}}{R_{\mathrm{w}}^{2}}\right)$

where $f_{\mathrm{p}}$ is the peak abundance, $R_{\mathrm{p}}$ is the radial distance of the peak of the distribution from the centre of the star, and $R_{\mathrm{w}}$, is the width of the shell at the $e$-folding radius. Using a shell distribution for both IK Tau and R Cas rather than a central Gaussian distribution significantly improved the $\chi^{2}$ fits of the models.

Similarly, we can firmly rule out a centrally-peaked model for TX Cam, as for such a model to fit the archival data we would expect conclusive detections in the HIFI data. As the undetected HIFI lines are of higher energy than the archival detections, a lower abundance in the inner regions of the CSE is expected, than in the outer regions, which points to a shell-like abundance distribution.

\subsubsection{SO}

For the radiative transfer analysis of SO we include 182 rotational energy levels, denoted $N_{J}$, up to $N=30$ in the ground and first excited vibrational states. There are 907 radiative transitions. These include pure rotational transitions in the $X^{3} \Sigma^{-}$ $v=0$ and $v=1$ states as well as the $v=1 \rightarrow 0$ rovibrational lines. There are 8629 collisional transitions including collisions between all rotational states within a vibrational state, as well as between vibrational states. The rotational energy levels, transition frequencies, and A-values have been adapted directly from the CDMS (Müller et al. 2001, 2005). The infrared line list has been computed directly from the rotational levels with the band-head frequency adjusted to give very good agreement with the line positions measured by Burkholder et al. (1987). The vibration-rotation line strengths have been computed in intermediate coupling and have been verified by comparison with the pure rotational line strengths in the CDMS tables. The vibration-rotation transition dipole moment has been taken to be 0.08843 Debye, which yields inverse lifetimes of 


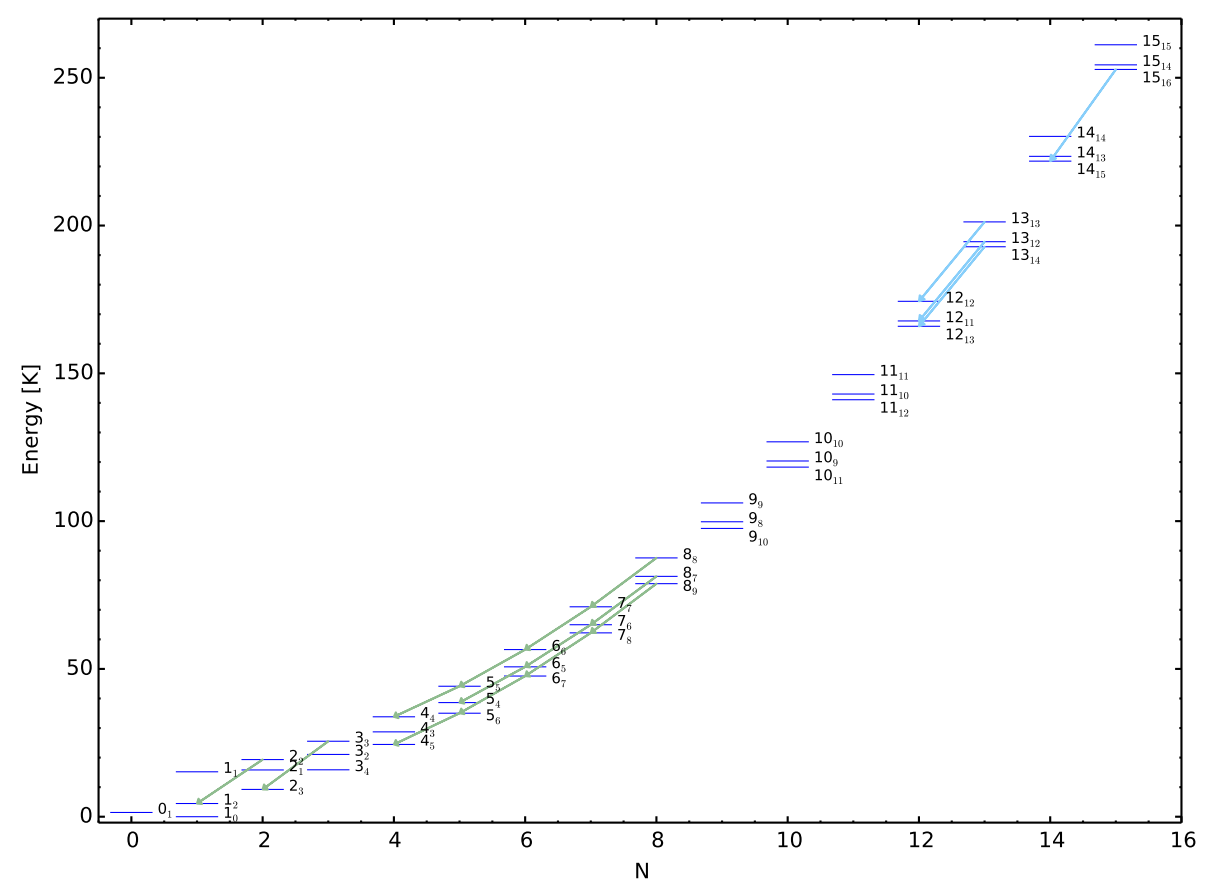

Fig. 1. SO energy level diagram with levels labelled using the $N_{J}$ convention. Transitions detected with HIFI and APEX towards R Dor are indicated in light blue and green, respectively.
$A_{\text {tot }}=3.6 \mathrm{~s}^{-1}$ for the $v=1 \rightarrow 0$ band as computed by Peterson $\&$ Woods (1990). The collisional rate coefficients for pure rotational transitions were adapted from the He-SO rates computed by Lique et al. (2006) with mass-scaling to $\mathrm{H}_{2}$ as in the smaller data set in the LAMDA database (Schöier et al. 2005). Rates for transitions within $v=1$ were assumed to be identical to those within $v=0$. Crude collision rates for $v=1 \rightarrow 0$ were scaled in proportion to normalised radiative line strengths for electricdipole-allowed transitions, with the largest values of the order of $1 \times 10^{-11} \mathrm{~cm}^{3} \mathrm{~s}^{-1}$.

In Fig. 1 we include an energy level diagram for SO. Here we have indicated all the transitions of SO detected towards R Dor with HIFI and APEX. These cover most of the transitions also detected in IK Tau, R Cas, W Hya, and TX Cam.

For the purposes of modelling the ${ }^{34} \mathrm{SO}$ emission in R Dor, we used a simpler molecular description than that for ${ }^{32} \mathrm{SO}$, including the rotational energy levels up to $N=30$, corresponding to those included for ${ }^{32} \mathrm{SO}$, but only in the ground vibrational state. When adopting the corresponding simpler molecular description for ${ }^{32} \mathrm{SO}$ in the case of R Dor specifically, we found that the final best fit model only shifted by a few percent between the detailed and simpler descriptions, justifying this approach for ${ }^{34} \mathrm{SO}$. There was, however, some shift in final model for the other, especially higher mass-loss rate, stars when changing between the detailed and simpler molecular description for $\mathrm{SO}$.

\subsection{2. $\mathrm{SO}_{2}$}

Our radiative transfer analysis of $\mathrm{SO}_{2}$ includes 2600 energy levels, denoted $J_{K a, K c}$, across the ground vibrational state and the $v_{1}=1(8.7 \mu \mathrm{m}), v_{2}=1(19.3 \mu \mathrm{m})$ and $v_{3}=1(7.3 \mu \mathrm{m})$ vibrationally excited states. Levels with energies up to $4830 \mathrm{~K}$ and $J=38$ were included. This gives 15243 radiative transitions, with spectroscopic data taken from the HITRAN database (Rothman et al. 2013), and 15244 collisional transitions. The collision rates in the literature for $\mathrm{SO}_{2}$ are inadequate for our purposes. Green (1995) calculated rate coefficients for $\mathrm{He}-\mathrm{SO}_{2}$ collisions in the infinite-order sudden approximation for the lowest 50 rotational levels (up to $100 \mathrm{~K}$ excitation energy and $J \leq 13$ only). Cernicharo et al. (2011) published rates for $\mathrm{H}_{2}-\mathrm{SO}_{2}$ collisions for the lowest 31 rotational levels at low temperatures, 5 to $30 \mathrm{~K}$. The rates for $\mathrm{H}_{2}$ impact were found to be approximately 10 times higher than corresponding rates for He impact. For the much larger number of states in our models, we adopted instead a set of crude collision rates in which the downward rate coefficient is proportional to the radiative line strength and normalised to a total collisional quenching rate of $2.0 \times 10^{-10} \mathrm{~cm}^{3} \mathrm{~s}^{-1}$, which is comparable to the highest collision rates found by Cernicharo et al. (2011). We tested the impact of the chosen collisional transition rates by multiplying the rates, in stages, by up to two orders of magnitude in both directions. We find that such drastic changes had only a very small and barely detectable effect on the resulting models. Hence we conclude that $\mathrm{SO}_{2}$ excitation is radiatively dominated with the choice of collisional transition rates playing only a minor role in the radiative transfer modelling.

In Fig. 2 we include an energy level diagram for $\mathrm{SO}_{2}$. Here we have indicated all transitions of $\mathrm{SO}_{2}$ detected towards $\mathrm{R}$ Dor with HIFI and APEX. As can be seen, $\mathrm{SO}_{2}$ has many close energy levels. This leads to a multitude of overlapping transitions, especially in AGB winds with typical expansion velocities of 5-25 km s${ }^{-1}$. The number of levels, transitions and overlaps presents some computational challenges, especially when it comes to fully taking overlapping lines into account or running exhaustive grids. To reduce running time to a manageable interval we restrict the overlaps so that only those within the sampled frequency range, between $200-1200 \mathrm{GHz}$, are included. This reduced the total number of overlaps by more than an order of magnitude (down to 441 lines participating in overlaps), hence decreasing running time and memory usage. This, however, neglects possible overlaps in pumping lines, which could have a significant effect on some of the lines included in the model. From what tests we were able to run we believe that the overall impact of these omitted lines is relatively minor. 
T. Danilovich et al.: Sulphur molecules in the circumstellar envelopes of M-type AGB stars

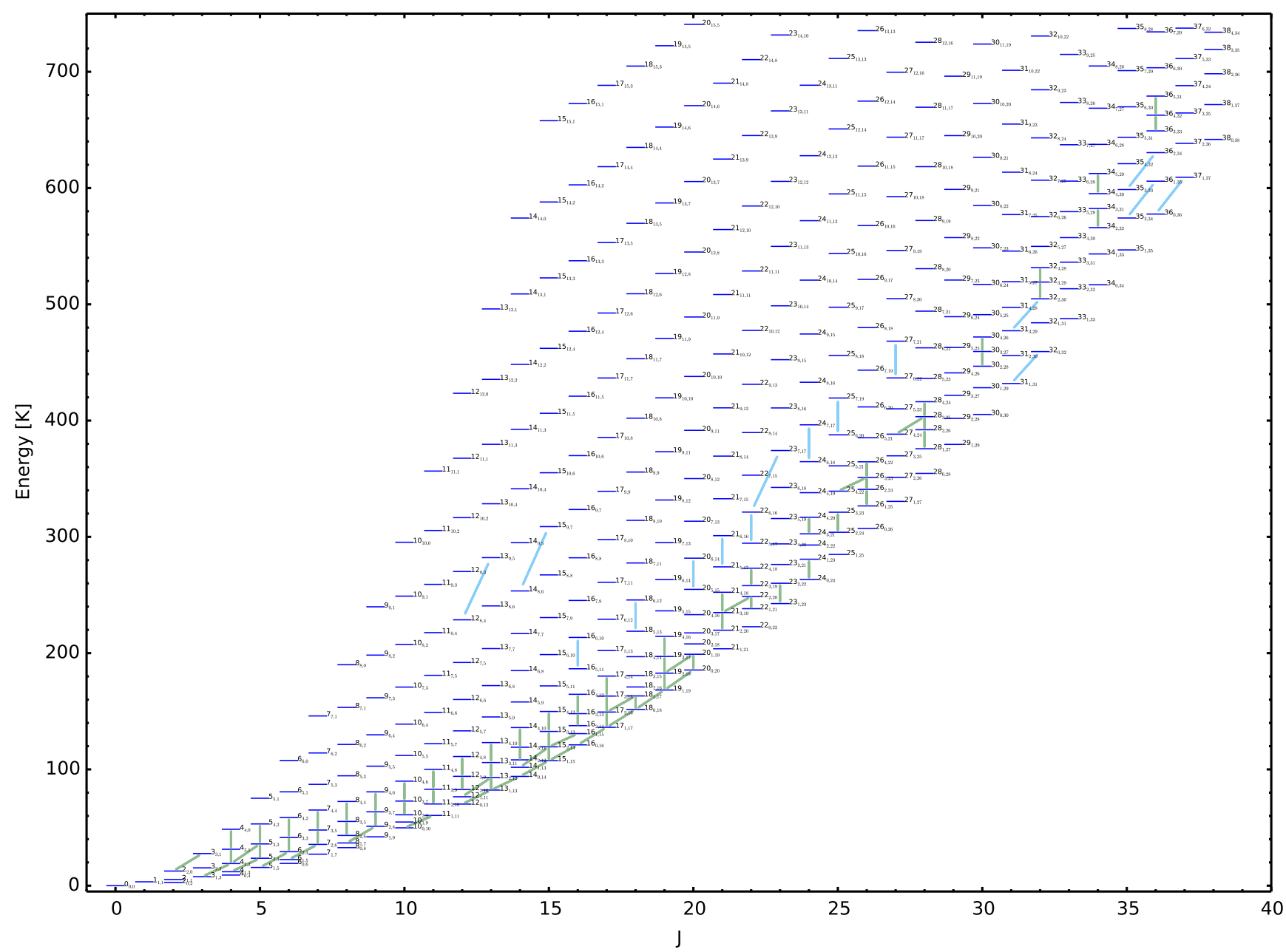

Fig. 2. $\mathrm{SO}_{2}$ energy level diagram. Levels are labelled $J_{K_{\mathrm{a}}, K_{\mathrm{c}}}$. Transitions detected with HIFI and APEX towards R Dor are indicated in light blue and green, respectively.

\section{2. $R$ Dor}

Our modelling is based on the radiative transfer results obtained by Maercker et al. (in prep) for CO in R Dor. They find a massloss rate of $\dot{M}=1.6 \times 10^{-7} \quad M_{\odot} \mathrm{yr}^{-1}$ and an expansion velocity of $v_{\infty}=5.7 \mathrm{~km} \mathrm{~s}^{-1}$. They also find an expansion velocity profile following

$v(r)=v_{\min }+\left(v_{\infty}-v_{\min }\right)\left(1-\frac{R_{\text {in }}}{r}\right)^{\beta}$

where $v_{\min }=3 \mathrm{~km} \mathrm{~s}^{-1}$ is taken to be the sound speed at $R_{\text {in }}=$ $1.6 \times 10^{14} \mathrm{~cm}$, the dust condensation radius. $\beta=1.5$ governs the acceleration of the gas, having the most significant impact in the inner regions, and hence on the excitation of the higher-energy lines. The other relevant stellar properties of R Dor are listed in Table 4.

\subsubsection{SO results}

To model the 17 SO lines detected towards R Dor with APEX and HIFI, we set up a grid sampling different $S O$ abundances and $e$-folding radii. We then ran a finer grid with steps of $0.1 \times 10^{-6}$ in abundance and $0.1 \times 10^{15} \mathrm{~cm}$ in $e$-folding radius to find the best possible fit to the observations. The results of our $\chi^{2}$ analysis can be seen in Fig. 3. Our resulting best-fit model, with $\chi_{\text {red }}^{2}=0.90$, has a peak $\mathrm{SO}$ abundance relative to $\mathrm{H}_{2}$ of $(6.7 \pm 0.9) \times 10^{-6}$ and $e$-folding radius $R_{e}=(1.4 \pm 0.2) \times 10^{15} \mathrm{~cm}$ and is plotted against the observed lines with respect to the LSR velocity in Fig. 4. A plot illustrating the goodness-of-fit for all the lines is given in Fig. 5. The abundance profile for SO is plotted in Fig. 8 along with $\mathrm{SO}_{2}$ and the $\mathrm{CO}$ and $\mathrm{H}_{2} \mathrm{O}$ results from Maercker et al. (in prep.) for comparison.

One of the detected SO lines, $\left(8_{9} \rightarrow 7_{8}\right)$, overlaps with $\mathrm{SO}_{2}\left(16_{4,12} \rightarrow 16_{3,13}\right)$ in its wing. We note that this is the only SO line which is significantly over-predicted by the model. Our code is unable to properly take heteromolecular overlaps such as this into account. We suspect that although the $\mathrm{SO}_{2}$ line is much fainter than the SO line (in fact it is difficult to see even in Fig. A. 1 where the $\mathrm{SO}_{2}$ model is overplotted), their interaction likely affects the flux from $\mathrm{SO}\left(8_{9} \rightarrow 78\right)$.

\subsection{2. $\mathrm{SO}_{2}$ results}

We detected $100 \mathrm{SO}_{2}$ lines in R Dor with APEX and HIFI. We exclude the $v_{2}=1\left(25_{4,22} \rightarrow 26_{1,25}\right)$ line at $279.497 \mathrm{GHz}$ from our analysis since it is most likely a maser ${ }^{4}$. We also concluded that it was not computationally viable to model the line with the

4 The main evidence for this supposition is that it in a vibrationally excited state, and that $\Delta K_{\mathrm{a}, \mathrm{c}}=3$ for this transition. Although this is an allowed transition, it is a very unlikely one under normal circumstances and, if included, our (non-masering) model predicts almost no emission from this transition. 

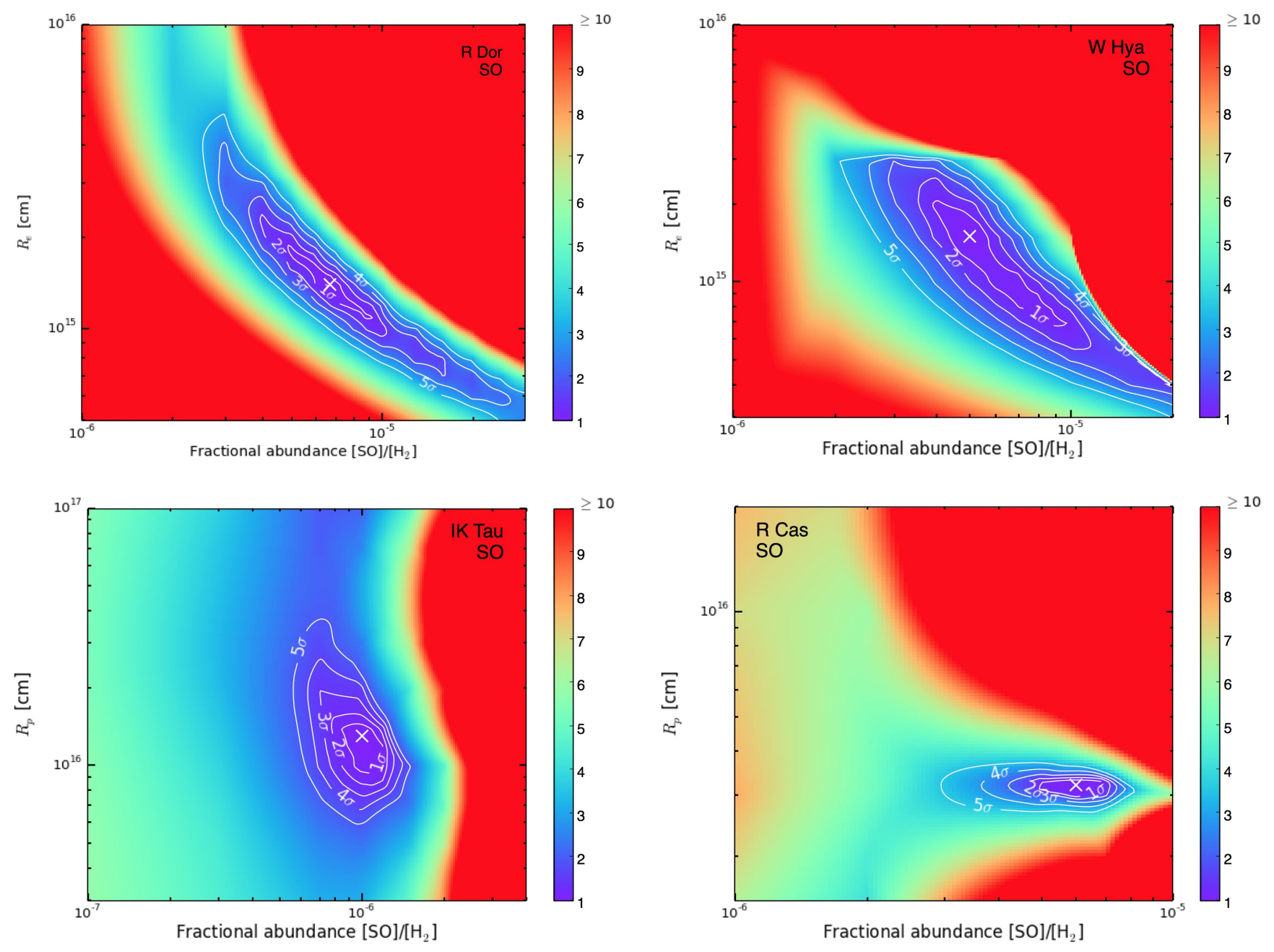

Fig. 3. SO $\chi^{2}$ plots for R Dor, W Hya, IK Tau and R Cas. The contours show the confidence intervals and the shading represents the $\chi_{\text {red }}^{2}$ value for the corresponding model, with the colour-bar indicating multiples of the minimum $\chi_{\text {red }}^{2}$ value. The white cross indicates our best-fit model (see Table 6). For IK Tau, the slice for which $R_{\mathrm{w}}=1.8 R_{\mathrm{p}}$ is shown. For R Cas, the slice for which $R_{\mathrm{w}}=1.0 R_{\mathrm{p}}$ is shown.

highest energy level in the ground state, $\left(40_{4,36} \rightarrow 40_{3,37}\right)$ at $341.403 \mathrm{GHz}$, as the number of additional levels and transitions required to fully account for this line represented a significant increase in computation time. (We would have required 3583 levels and 19889 radiative transitions.) The two excluded lines are plotted in Fig. 6.

This leaves us with 98 detected $\mathrm{SO}_{2}$ lines with which to constrain our model. Our best fit model has $f_{\mathrm{p}}=5.0 \times 10^{-6}$, $R_{e}=1.6 \times 10^{15} \mathrm{~cm}$ and $\chi_{\text {red }}^{2}=3.7$. Due to the significant computational time in running $\mathrm{SO}_{2}$ models, we are unable to provide a comprehensive error analysis as we do for the SO model, hence the lack of formal uncertainties on our results. The model lines are plotted with the observed lines in Fig. A.1 with goodness of fit shown in Fig. 7. Overlaps are discussed in detail in Sect. 3.2.3. Figure 8 shows our best-fit abundance profiles for $\mathrm{SO}_{2}$ and $\mathrm{SO}$, along with the results for $\mathrm{CO}$ and $\mathrm{H}_{2} \mathrm{O}$ from Maercker et al. (in prep.).

There is a lot of scatter in the goodness-of-fit plots in Fig. 7. There is no trend in goodness-of-fit with upper energy level or $J$, but the observed lines that are most strongly under-predicted by the model are those lines for which the upper energy level has quantum number $K_{\mathrm{a}} \geq 6$ (see lower right plot in Fig. 7). This corresponds to the lines further away from the "backbone" of $K_{\mathrm{a}}=0,1$ energy levels in the energy level diagram in Fig. 2. We suspect this could be partially due to our exclusion of overlaps for lines outside of the observed frequency range (see Sect. 3.1.2). When testing models with and without overlaps enabled, we note that some lines that do not participate in overlaps can still be strongly affected by the inclusion (or not) of overlaps in our model. For example $\mathrm{SO}_{2}\left(27_{7,21} \rightarrow 27_{6,22}\right)$ at $657.885 \mathrm{GHz}$ was one such line, with the model predicting weaker emission by a factor of a few when overlaps were omitted. Unfortunately, due to computational limitations, it is not feasible to properly include overlaps in a full radiative transfer analysis, as discussed in Sect. 3.1.2. It should also be noted that the $\mathrm{R}$ Dor data were taken over a long observational campaign (see Sect. 2), so any variability in $\mathrm{SO}_{2}$ line brightnesses with pulsation period may contribute to the scatter.

\subsubsection{Overlapping lines}

Table 5 contains an inventory of known line overlaps for the presented lines. In our radiative transfer modelling, we are able to take into account overlaps which occur between two lines of the same molecule - i.e. two $\mathrm{SO}_{2}$ lines. (For computational purposes we only include $\mathrm{SO}_{2}$ overlaps in the range $200 \mathrm{GHz}-1.2 \mathrm{THz}$. 
T. Danilovich et al.: Sulphur molecules in the circumstellar envelopes of M-type AGB stars
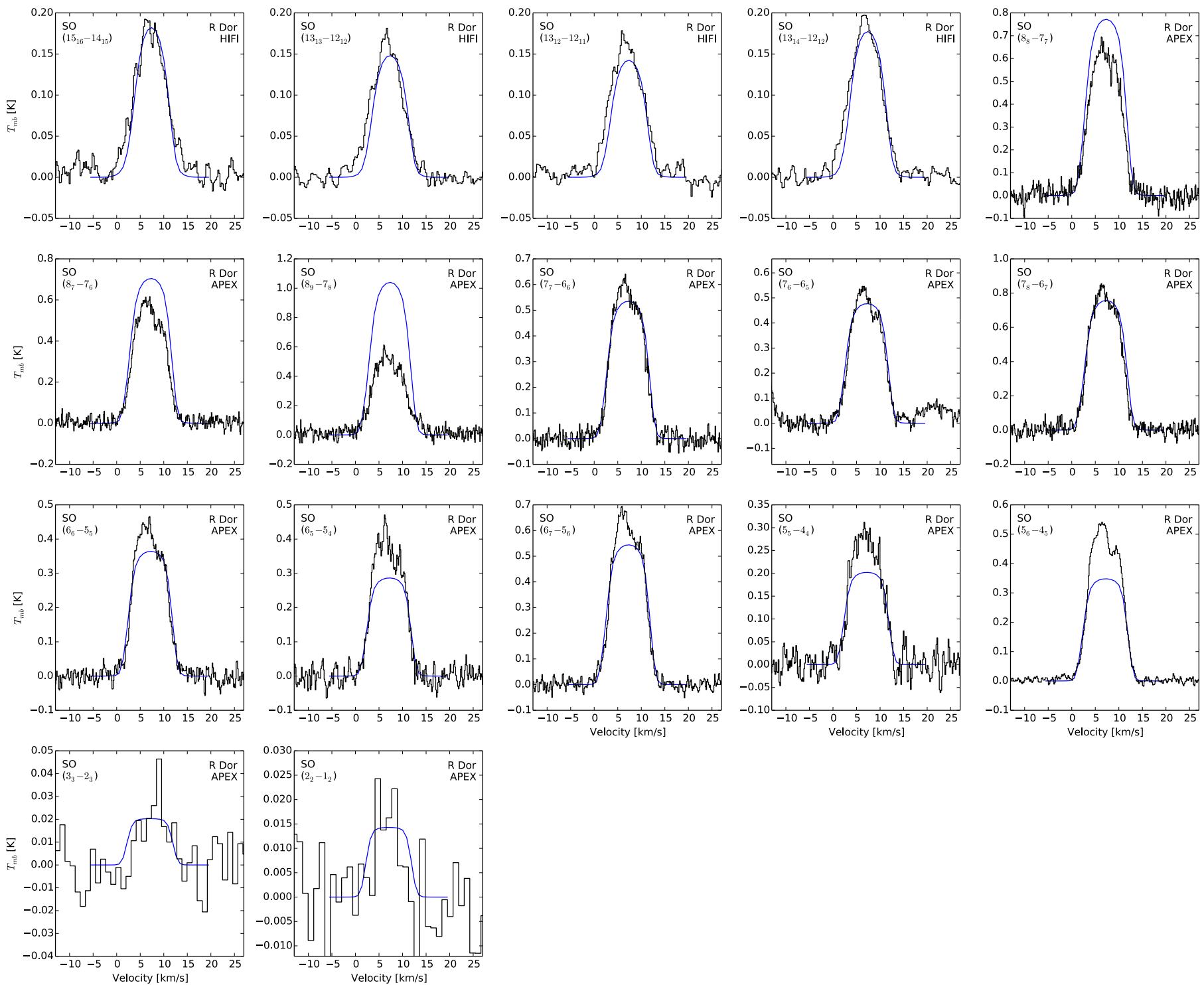

Fig. 4. SO models (blue lines) and observations (black histograms) for R Dor.

Table 5. Overlapping lines in R Dor.

\begin{tabular}{lllll}
\hline \hline Primary line & Frequency & Secondary line & Frequency & Notes \\
\hline $\mathrm{SO}_{2} 32_{0,32} \rightarrow 31_{1,31}$ & 571.553 & $\mathrm{SO}_{2} 32_{2,30} \rightarrow 31_{3,29}$ & 571.532 & Two distinct peaks \\
$\mathrm{SO}_{2} 13_{1,13} \rightarrow 12_{0,12}$ & 251.200 & $\mathrm{SO}_{2} 8_{3,5} \rightarrow 8_{2,6}$ & 251.211 & Two distinct peaks \\
$\mathrm{SO}_{9} \rightarrow 78$ & 346.528 & $\mathrm{SO}_{2} 16_{4,12} \rightarrow 16_{3,13}$ & 346.524 & SO line strongly dominates, SO line in $\mathrm{SO}_{2}$ wing \\
$\mathrm{SO}_{2} 24_{7,17} \rightarrow 26_{6,18}$ & 659.898 & $\mathrm{SO}_{2} 40_{1,39} \rightarrow 40_{0,40}$ & 659.886 & Primary line dominates, secondary appears in wing \\
$\mathrm{SO}_{2} 6_{3,3} \rightarrow 6_{2,4}$ & 254.281 & $\mathrm{SO}_{2} 24_{2,22} \rightarrow 24_{1,23}$ & 254.283 & Unresolved overlap of two lines of similar strength \\
$\mathrm{SO}_{2} 32_{3,29} \rightarrow 32_{2,30}$ & 300.273 & $\mathrm{SO}_{2} 24_{8,16} \rightarrow 25_{7,19}\left(v_{2}=1\right)$ & 300.280 & Secondary line not detected \\
$\mathrm{SO}_{2} 15_{4,12} \rightarrow 15_{3,13}$ & 357.241 & $\mathrm{SO}_{2} 37_{4,34} \rightarrow 38_{1,37}\left(v_{2}=1\right)$ & 357.230 & Secondary line not detected \\
$\mathrm{SO}_{2} 12_{3,9} \rightarrow 12_{2,10}$ & 237.069 & $\mathrm{SO}_{2} 26_{3,23} \rightarrow 25_{4,22}\left(v_{2}=1\right)$ & 237.062 & Secondary line not detected \\
$\mathrm{SO}_{2} 7_{3,5} \rightarrow 7_{2,6}$ & 257.100 & $\mathrm{SO}_{2} 8_{3,5} \rightarrow 8_{2,6}\left(v_{2}=1\right)$ & 257.099 & Lines coincide very closely; not distinguishable \\
$\mathrm{SO}_{2} 13_{2,12} \rightarrow 12_{1,11}$ & 345.339 & $\mathrm{H}^{13} \mathrm{CN} 4 \rightarrow 3$ & 345.340 & Lines not distinguishable in profile \\
\hline
\end{tabular}

Notes. ${ }^{(*)}$ The $\mathrm{SO}_{2}\left(40_{1,39} \rightarrow 40_{0,40}\right)$ line is not included in our final model. See discussion in Sect. 3.2.3.

Note, however, that all possible homomolecular overlaps are taken into account for SO in all modelled stars.) However, if there is a line overlap between two lines generated by different molecules, we are unable to properly treat this, as our code only allows for the modelling of one molecular species at a time. In R Dor we observe two such heteromolecular overlaps. The first between the $\mathrm{SO}\left(8_{9} \rightarrow 7_{8}\right)$ and $\mathrm{SO}_{2}\left(16_{4,12} \rightarrow 16_{3,13}\right)$ lines, where the much weaker $\mathrm{SO}_{2}$ line appears in the wing of the bright SO line, and the second between $\mathrm{SO}_{2}\left(13_{2,12} \rightarrow 12_{1,11}\right)$ and $\mathrm{H}^{13} \mathrm{CN}(4 \rightarrow 3)$, where the two lines coincide very closely so as to be indistinguishable. Based on our model, we expect approximately half the flux to be due to the $\mathrm{H}^{13} \mathrm{CN}(4 \rightarrow 3)$ transition, 

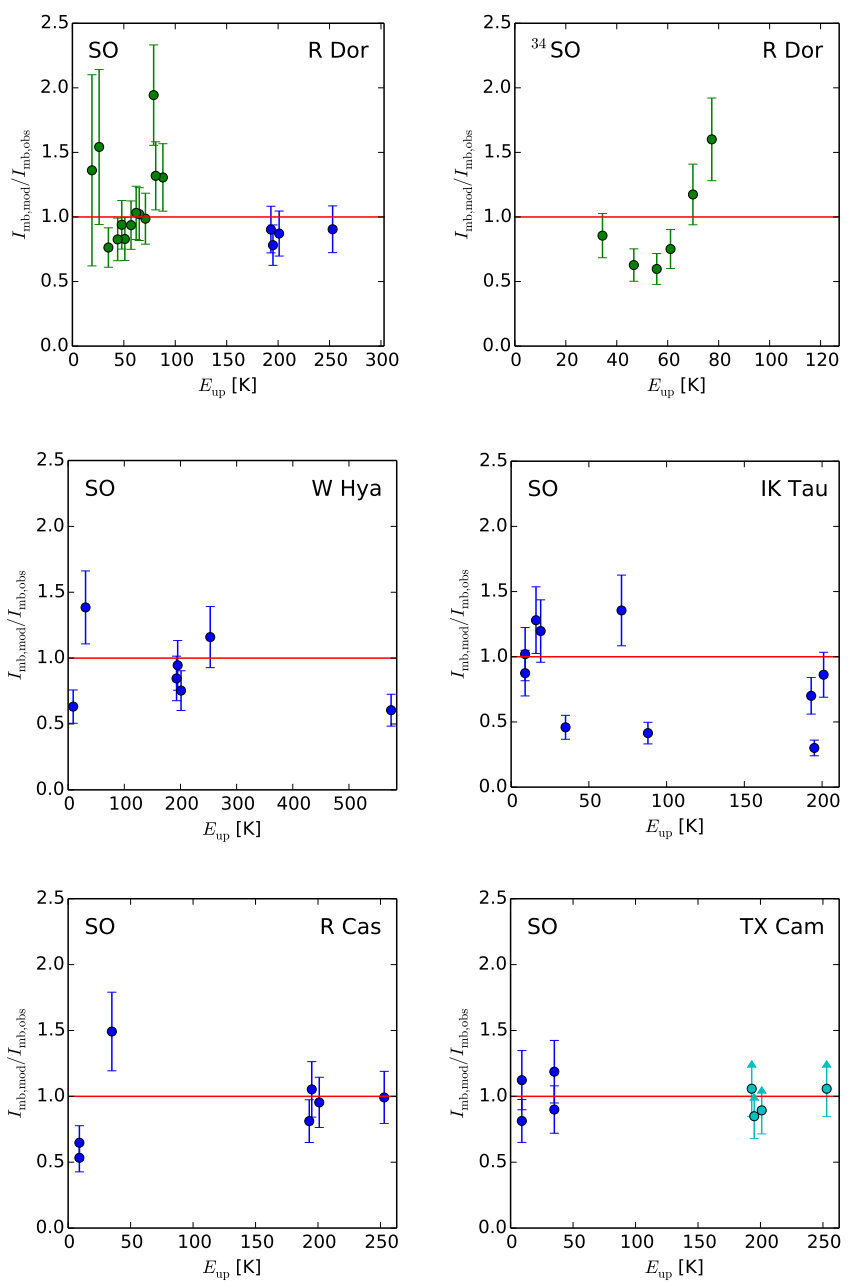

Fig. 5. SO goodness of fit plots for R Dor, R Cas, IK Tau, and W Hya. New HIFI lines as well as archival data listed in Table C.3 are included. The green points in the $\mathrm{R}$ Dor plots represent the observations from the APEX spectral survey. Undetected HIFI lines are shown as cyan points with arrows, in this case representing lower limits because the vertical axis is the ratio of model integrated intensities to observed integrated intensities or the upper limits thereof.
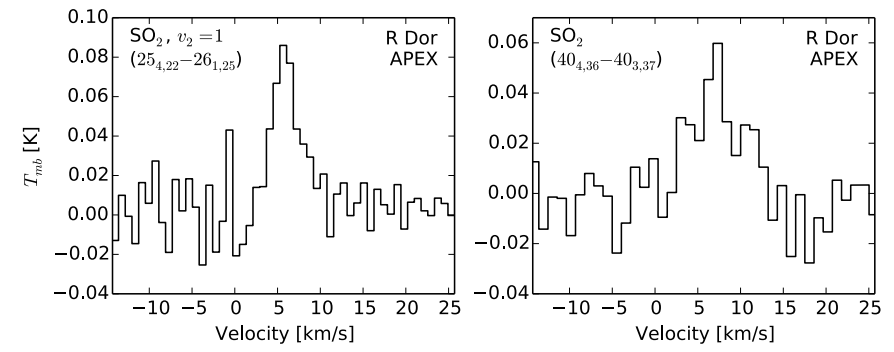

Fig. 6. $\mathrm{SO}_{2}$ lines excluded from modelling for R Dor. See text for full explanation.

which would agree with the $\mathrm{H}^{13} \mathrm{CN}(3 \rightarrow 2)$ line also covered by the APEX survey. However, without modelling $\mathrm{H}^{13} \mathrm{CN}$, it is not possible to fully gauge the impact of this overlap on our model.

The remaining line overlaps for lines modelled in this paper are homomolecular.

Three of the line pairs that are treated as overlapping in the code consist of a bright primary line in the vibrational ground state and a very weak secondary line in the $v_{2}=1$ vibrationally excited state. As can be seen in Fig. A.1, these secondary lines
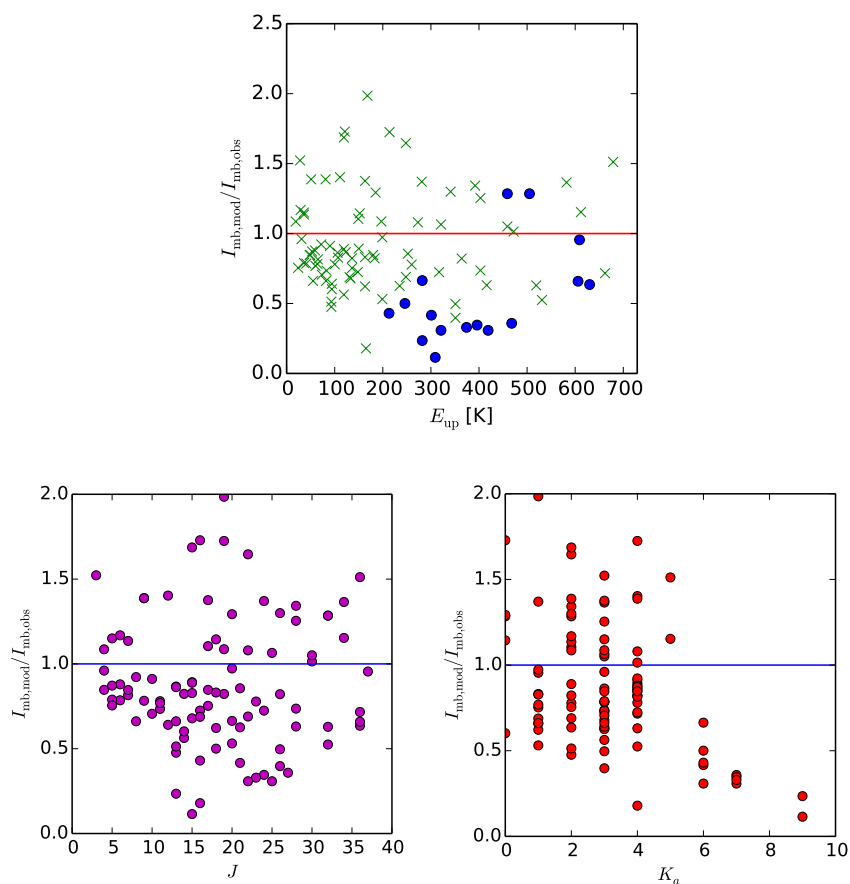

Fig. 7. $\mathrm{SO}_{2}$ goodness of fit plots for R Dor. Top: goodness of fit with upper energy level of the transition. HIFI lines are shown as blue points and APEX lines are shown as green crosses. Error bars are excluded to make the plot clearer to read. Lower left: goodness of fit with $J$. Lower right: goodness of fit with $K_{\mathrm{a}}$, a clear downwards trend for $K_{\mathrm{a}} \geq 6$.

are not detectable above the noise in our observations, but are taken into account in our modelling.

The $\mathrm{SO}_{2}\left(24_{7,17} \rightarrow 26_{6,18}\right)$ line at $659.898 \mathrm{GHz}$ overlaps with the $\mathrm{SO}_{2}\left(40_{1,39} \rightarrow 40_{0,40}\right)$ at $659.886 \mathrm{GHz}$ and we would expect the latter to have an effect on the former. However, the $\mathrm{SO}_{2}$ $\left(40_{1,39} \rightarrow 40_{0,40}\right)$ line falls outside of the range of energy levels we included in our model. As noted in Sect. 3.1.2, it was not feasible to include a larger number of higher energy levels, hence this particular overlap is not taken into account in our modelling.

\subsubsection{Isotopologue results}

Based on the analysis of $6{ }^{34} \mathrm{SO}$ lines, and assuming the same $e$-folding radius as found for ${ }^{32} \mathrm{SO}$, we find a ${ }^{34} \mathrm{SO}$ abundance of $(3.1 \pm 0.8) \times 10^{-7}$ in a best fit model that has $\chi_{\text {red }}^{2}=1.4$. This gives a ${ }^{32} \mathrm{SO} /{ }^{34} \mathrm{SO}$ ratio of $21.6 \pm 8.5$. The best fit model is shown in Fig. 9. The goodness of fit plot showing the ratio between the model and observed integrated intensities is shown in Fig. 5.

Modelling ${ }^{34} \mathrm{SO}_{2}$ in the same detailed manner as we have modelled ${ }^{32} \mathrm{SO}_{2}$ is impractical given the computational time required, the complexity of the molecular data file, and the low number of detected lines. However, all of the ${ }^{32} \mathrm{SO}_{2}$ lines we modelled are optically thin, so we can approximate the ${ }^{32} \mathrm{SO}_{2} /{ }^{34} \mathrm{SO}_{2}$ ratio by comparing the intensity ratios of two lines of the same transition. The best ${ }^{34} \mathrm{SO}_{2}$ transition for this purpose is $20_{0,20} \rightarrow 19_{1,19}$. Comparing the integrated intensities for this transition, we find a ${ }^{32} \mathrm{SO}_{2} /{ }^{34} \mathrm{SO}_{2}$ ratio of $21.6 \pm 12.1$, in good agreement with the result from ${ }^{34} \mathrm{SO}$ modelling.

The solar system value of ${ }^{32} \mathrm{~S} /{ }^{34} \mathrm{~S}$ is 22.5 (Cameron 1973) and Kahane et al. (1988) found a value of 20.2 for the carbon star CW Leo using SiS isotopologues, both in agreement with our results. 

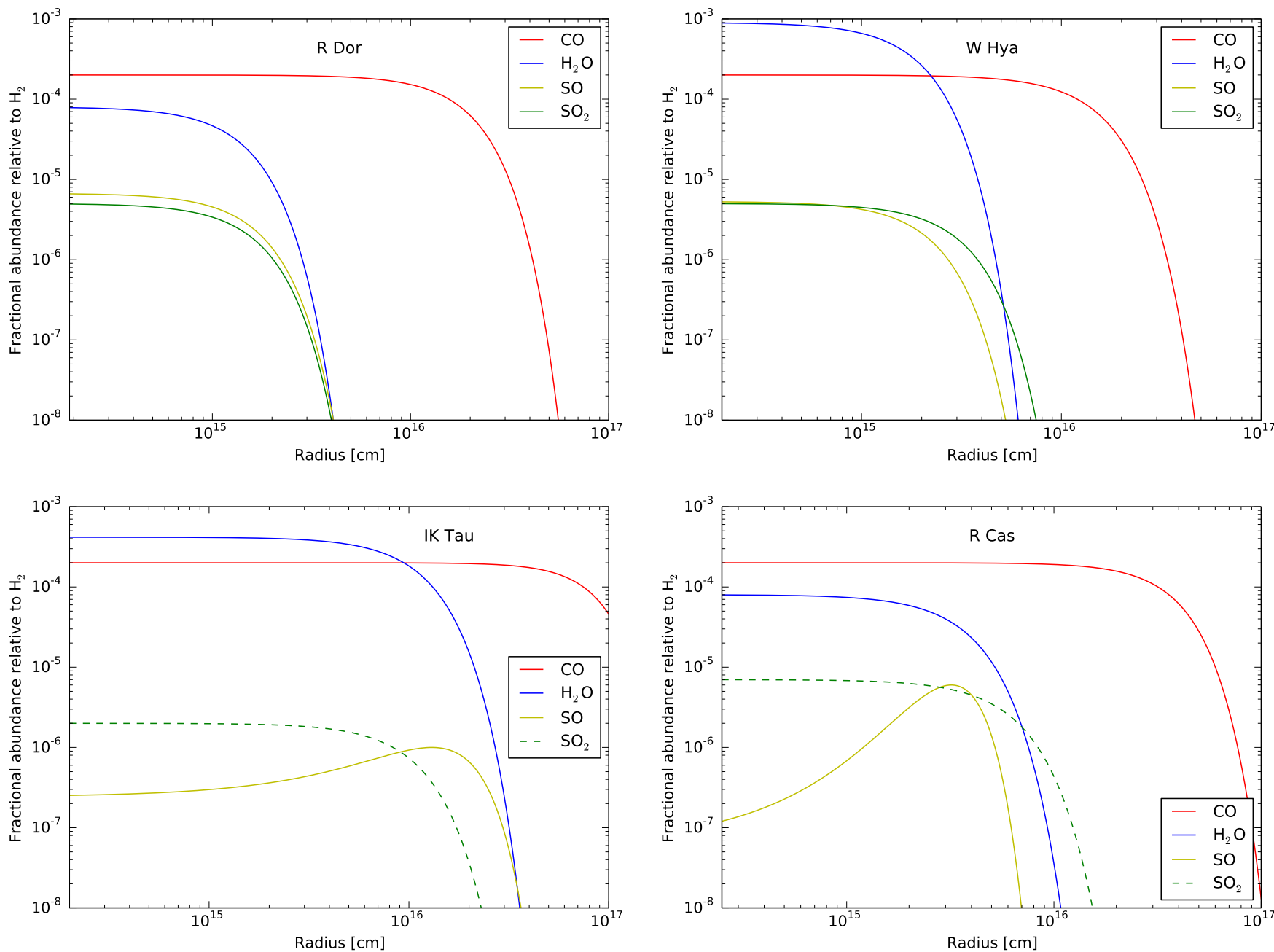

Fig. 8. Abundance profiles for R Dor, W Hya, IK Tau and R Cas. The abundances for $\mathrm{CO}$ and $\mathrm{H}_{2} \mathrm{O}$ are taken from Maercker et al. (in prep.), except for W Hya, for which they are taken from Khouri et al. (2014a,b). The dashed line for the $\mathrm{SO}_{2}$ results for IK Tau and R Cas indicates that they are tentative.

While a detailed model of ${ }^{34} \mathrm{SO}_{2}$ would be extremely time consuming, a detailed model of $\mathrm{SO}^{18} \mathrm{O}$ would not be computationally feasible. Due to the asymmetry of the two oxygen atoms, $\mathrm{SO}^{18} \mathrm{O}$ has approximately double the number of energy levels and transitions as $\mathrm{SO}_{2}$, when looking at the same energy range, meaning that an $\mathrm{SO}^{18} \mathrm{O}$ molecular data file would have to be approximately twice the size of our already very large $\mathrm{SO}_{2}$ file to probe a similar range of energies. The more complex energy level structure also means it is not possible to directly compare lines between $\mathrm{SO}^{18} \mathrm{O}$ and $\mathrm{SO}_{2}$, even when the transitions have the same quantum numbers. For ${ }^{34} \mathrm{SO}_{2}$ and $\mathrm{SO}^{18} \mathrm{O}$ we present the (tentative) detections in Fig. A.2.

\subsection{Other $M$ stars}

We model $\mathrm{SO}$ and $\mathrm{SO}_{2}$ line emission for the remaining stars using HIFI observations, as listed in Table C.2, and archival observations with different ground-based instruments, as listed in Table C.3. Several of these older observations probe energy levels significantly lower than the HIFI observations, allowing us to better constrain the size of the emitting molecular envelope. This is particularly important for IK Tau, where only the three $N=13 \rightarrow 12$ SO lines were detected with HIFI, as these are emitted from a similar region of the CSE.
The stellar parameters used in our $\mathrm{SO}$ and $\mathrm{SO}_{2}$ models, taken from CO model results, are listed in Table 4.

\subsubsection{W Hya}

In the case of W Hya we find an SO model that fits the data well using the Gaussian abundance distribution given in Eq. (3). We found $f_{\mathrm{p}}=(5.0 \pm 1.0) \times 10^{-6}$ and $R_{e}=(1.5 \pm 0.5) \times 10^{15} \mathrm{~cm}$, with $\chi_{\text {red }}^{2}=2.57$. This result is qualitatively similar to that of R Dor. As with R Dor, this suggests that SO in the CSE of W Hya is formed close to the star and is not found in a shell around the star as might be expected if it were a photodissociation product of another molecule such as $\mathrm{H}_{2} \mathrm{~S}$. The HIFI observations and model line plots for SO are shown in Fig. 10. The corresponding $\chi^{2}$ plot is shown in Fig. 3 .

The HIFI observations and model line plots for $\mathrm{SO}_{2}$ towards W Hya are shown in Fig. 11. The main difficulty we had in fitting an $\mathrm{SO}_{2}$ model was finding a model which fit the two highestenergy lines. As can be seen in Table C.2, the $\mathrm{SO}_{2}\left(37_{1,37} \rightarrow\right.$ $\left.36_{0,36}\right)$ and $\mathrm{SO}_{2}\left(36_{1,35} \rightarrow 35_{2,34}\right)$ lines are only $\sim 3 \mathrm{~K}$ apart in upper energy level. Also we note that the lower-energy line is almost a factor of 3 brighter than the higher-energy line. Our model invariably predicts a smaller difference in intensity with the higher-energy line being the brighter. The same is true for 

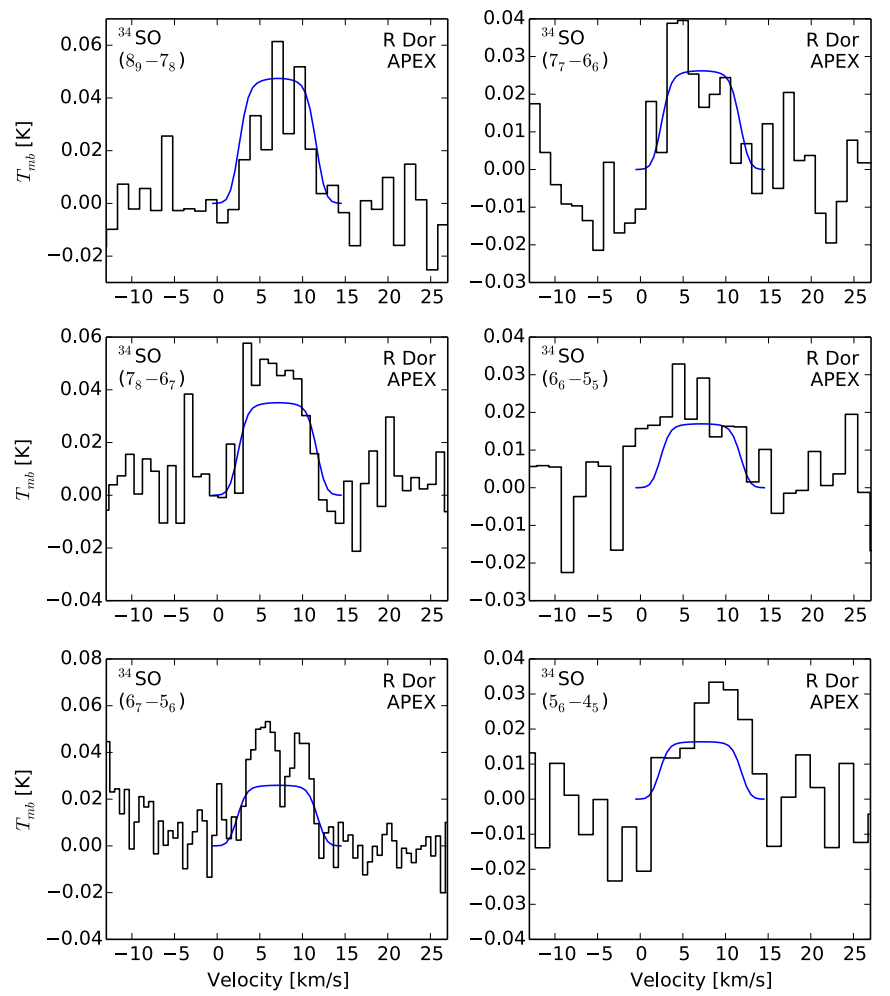

Fig. 9. ${ }^{34} \mathrm{SO}$ model (blue lines) and observations (black histograms) for R Dor.

R Dor, however, in R Dor the detected lines reflect this (although the model fit is not perfect). This phenomenon is probably due in part to the noise in our observations but could also reflect a problem with our molecular description of $\mathrm{SO}_{2}$. In this case, the most likely cause is the cut-off in included energy levels at $J=38$. The variation in these lines cannot be due to variations in brightness due to stellar pulsations as both lines were observed simultaneously (and, indeed, all the $\mathrm{SO}_{2}$ lines in $\mathrm{W}$ Hya were observed within two days). In any case, the apparently outlying line of $\left(37_{1,37} \rightarrow 36_{0,36}\right)$ strongly contributes to the poorly fitting model we find for $\mathrm{SO}_{2}$ in $\mathrm{W}$ Hya. We are able to find a better fit by excluding this line, but do not have a strong basis for doing so, hence we leave it in.

Our best fit model for $\mathrm{SO}_{2}$ has $f_{\mathrm{p}}=5.0 \times 10^{-6}$, based on a small grid with steps of $0.5 \times 10^{-6}$, and $R_{e}=3.0 \times 10^{15} \mathrm{~cm}$, based on a small grid with steps of $0.5 \times 10^{15} \mathrm{~cm}$. This model has $\chi_{\text {red }}^{2}=5.7$. We also test an $\mathrm{SO}_{2}$ model using the parameters we found for SO. That model is not a significantly worse fit with almost the same $\chi_{\text {red }}^{2}$.

The abundance distributions for $\mathrm{SO}$ and $\mathrm{SO}_{2}$, along with the $\mathrm{CO}$ and $\mathrm{H}_{2} \mathrm{O}$ abundance distributions from Khouri et al. (2014a,b) for comparison, are shown in Fig. 8.

\subsubsection{IK Tau}

When we try to fit the SO IK Tau observations with a centrally peaked Gaussian distribution, we cannot constrain the $e$-folding radius with the available data. The $\chi^{2}$ analyses of centrallypeaked Gaussian models point towards very large $e$-folding radii, significantly larger (by more than half an order of magnitude) than the half-abundance radius Maercker et al. (in prep.) found for the corresponding $\mathrm{CO}$ envelope. Since it is highly unlikely that the SO envelope is more extensive than that of $\mathrm{CO}$, we
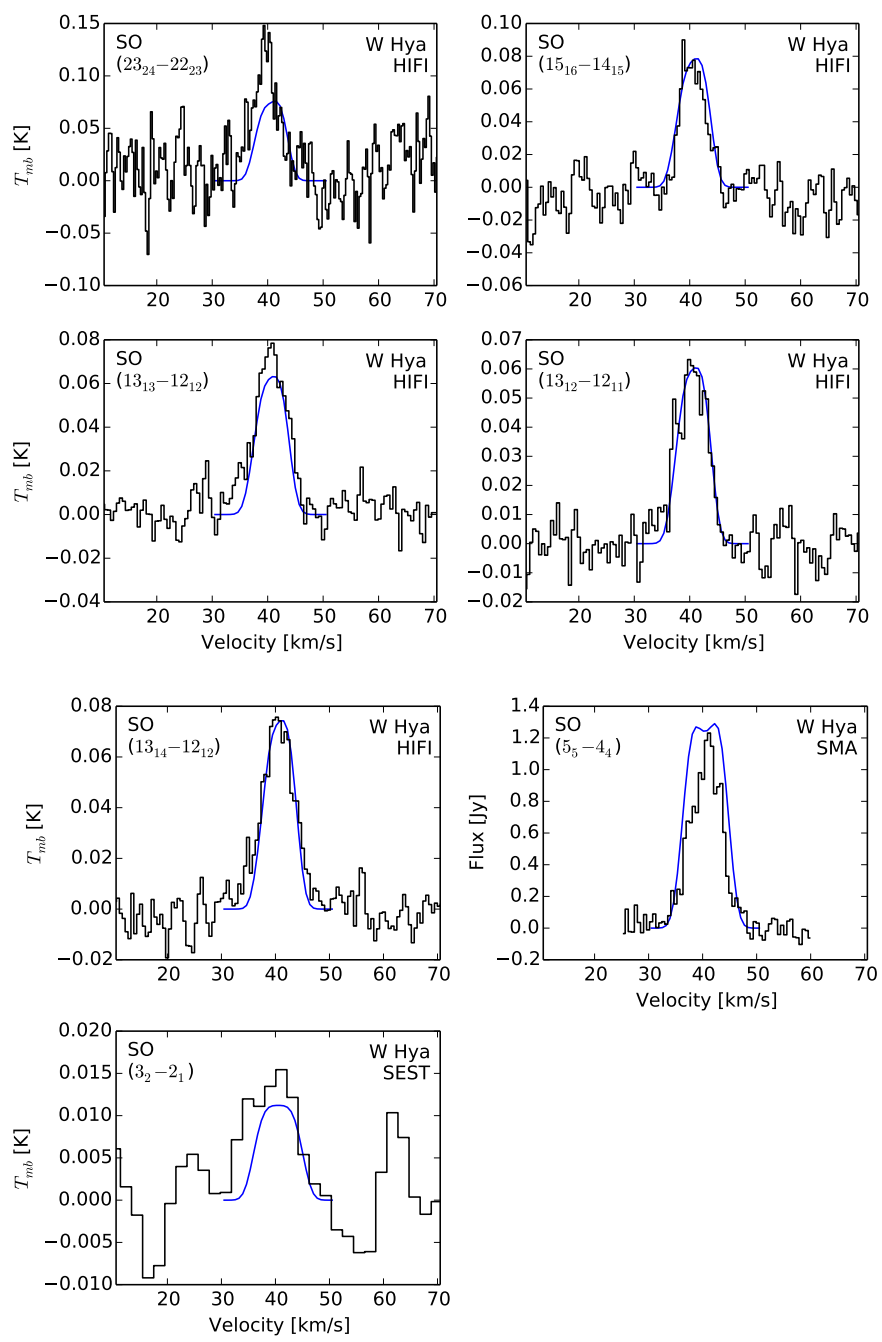

Fig. 10. Models (blue lines) and observations (black histograms) for SO towards W Hya.

conclude that a centrally-peaked Gaussian distribution is unlikely for SO in IK Tau. Instead, we run a three-parameter grid across $f_{\mathrm{p}}, R_{\mathrm{p}}$, and $R_{\mathrm{w}}$ (see Eq. (4)) to find the best model. We find $f_{\mathrm{p}}=(1.0 \pm 0.2) \times 10^{-6}, R_{\mathrm{p}}=(1.3 \pm 0.2) \times 10^{16} \mathrm{~cm}$, and $R_{\mathrm{w}}=1.8 R_{\mathrm{p}}$ (which we gridded in steps of $0.2 R_{\mathrm{p}}$ ), with $\chi_{\text {red }}^{2}=4.67$ and the resultant lines are shown in Fig. 12.

The $\chi^{2}$ plot for SO in IK Tau is shown in Fig. 3. IK Tau has a significantly larger $\chi^{2}$ value for the best fit model (compared with R Dor and W Hya) because of some noisy observations. This is also seen in the goodness of fit plot in Fig. 5. In comparison, R Dor and W Hya have brighter and more uniform line observations, making it easier to find a good model fit.

Decin et al. (2010a) perform a radiative transfer analysis of IK Tau in a way that is similar to our method. They find an SO abundance distribution that is similar to our shell-like distribution, but with an increased abundance in the inner region. They find an abundance at $200 R_{*}$ (which corresponds to about $3 \times 10^{15} \mathrm{~cm}$ ) of $\sim 2 \times 10^{-7}$ using two lines to fit the model. This did not change significantly in the follow up in Decin et al. (2010b) which included one of the HIFI lines as well. Our model results give a corresponding abundance about a factor of 2 higher at the same radius but using a different shape for the abundance 
T. Danilovich et al.: Sulphur molecules in the circumstellar envelopes of M-type AGB stars
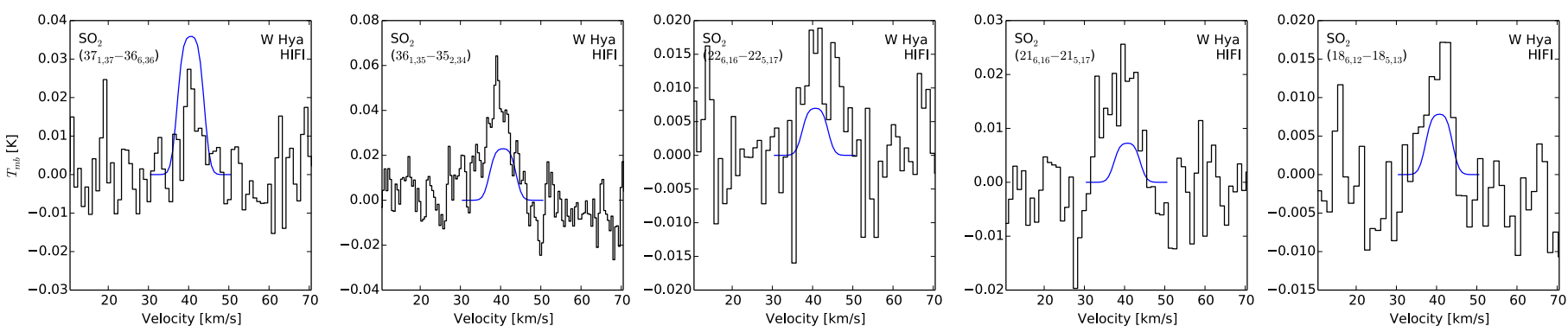

Fig. 11. Models (blue lines) and observations (black histograms) for $\mathrm{SO}_{2}$ towards W Hya.

distribution. We also use 10 lines with a broader range of energy levels to constrain the model.

In the case of $\mathrm{SO}_{2}$ in IK Tau we are unable to include overlaps as we do for R Dor and W Hya due to the larger expansion velocity of the circumstellar gas around IK Tau. The larger expansion velocity means there are a larger number of overlaps (since the lines are about three times wider than for R Dor) which quickly become computationally infeasible to fully account for.

We could not find a consistent model for IK Tau that matched all the available observed $\mathrm{SO}_{2}$ lines. In particular, there was a very large scatter in goodness-of-fit for the lines with upper energy levels of $136 \mathrm{~K}$ or less (which is all of the lines other than the one HIFI observation). There was no way to simultaneously fit all these observed lines well. A centrally-peaked Gaussian model matches the data reasonably well - particularly the HIFI line, which according to the best shell model should have been a non-detection - and much better than the shell model. A Gaussian model with $e$-folding radius located at the peak of the SO distribution is a better fit than a model with the SO distribution parameters, but we find that decreasing the $e$-folding radius to $R_{e}=1 \times 10^{16} \mathrm{~cm}$ gives a better fit again. We cannot constrain the $e$-folding radius better than by a factor of $\sim 2$, however, because of the large scatter in the lower-energy lines. The model we present in this paper, plotted in Fig. 13, has a peak $\mathrm{SO}_{2}$ abundance $f_{\mathrm{p}}=2 \times 10^{-6}$, and $R_{e}=1 \times 10^{16} \mathrm{~cm}$. This model has $\chi_{\text {red }}^{2}=18.4$, the high value reflecting the poor overall fit. The large scatter in the IK Tau $\mathrm{SO}_{2}$ lines could be due to variability in line brightness with pulsation period. The data we used were observed at different times corresponding to different phases of pulsation. For example, the brightest lines $\left(17_{1,17} \rightarrow 1_{0,16}\right)$ and $\left(13_{2,12} \rightarrow 12_{1,11}\right)$, were observed less than two weeks apart close to maximum brightness in 2006 . The most under-predicted line, $\left(14_{3,11} \rightarrow 14_{2,12}\right)$, was observed four months later when the star was approaching minimum brightness. On the other hand, the most well-fit lines - those with $J=5,4,3$ as can be seen in Fig. 13 - were variously taken close to minimum and maximum brightness, so perhaps it is the higher $J$ lines which are most strongly affected. Future monitoring of these lines observationally would allow us to confirm whether the effect on the higher- $J$ lines is really due to variability over a pulsation period.

The abundance distributions for $\mathrm{SO}$ and $\mathrm{SO}_{2}$ in IK Tau, along with the $\mathrm{CO}$ and $\mathrm{H}_{2} \mathrm{O}$ abundance distributions from Maercker et al. (in prep.) for comparison, are shown in Fig. 8. In general, we do not consider our $\mathrm{SO}_{2}$ results for IK Tau conclusive. A more rigorous model which is properly able to take overlaps into consideration and which perhaps includes more lines in the intermediate to high energy range (with upper energy level >136 K) is recommended.

Decin et al. (2010a) have similar issues modelling the $\mathrm{SO}_{2}$ in IK Tau, especially with the $\left(17_{1,17} \rightarrow 16_{0,16}\right)$ and $\left(13_{2,12} \rightarrow 12_{1,11}\right)$ lines which we also strongly under-predict, as
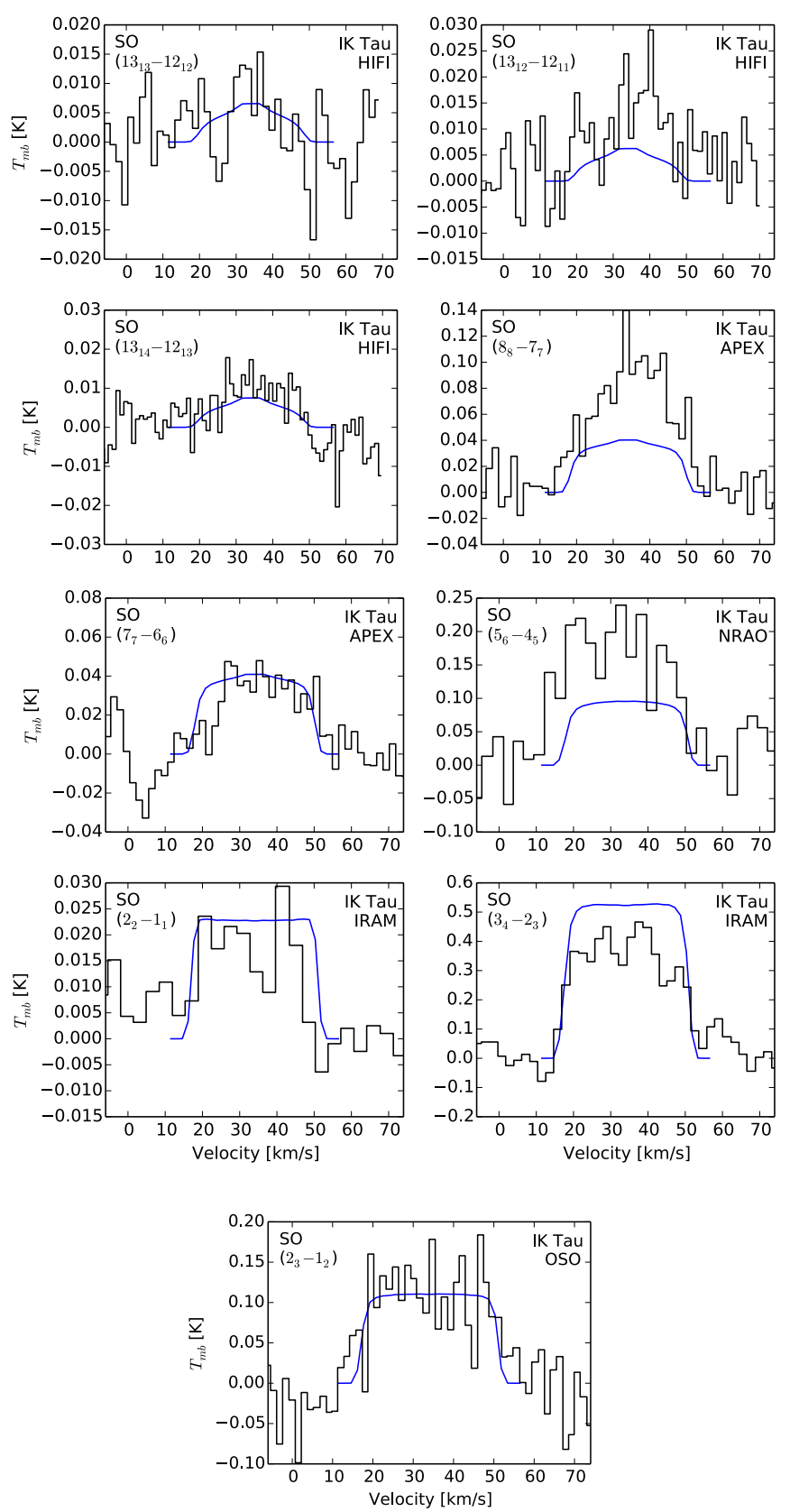

Fig. 12. SO models (blue lines) and observations (black histograms) for IK Tau.

can be seen in Fig. 13. When they exclude these two lines, Decin et al. (2010a) find a high inner abundance of $\mathrm{SO}_{2}$, in general agreement with our results. The poor fit of our model could be 

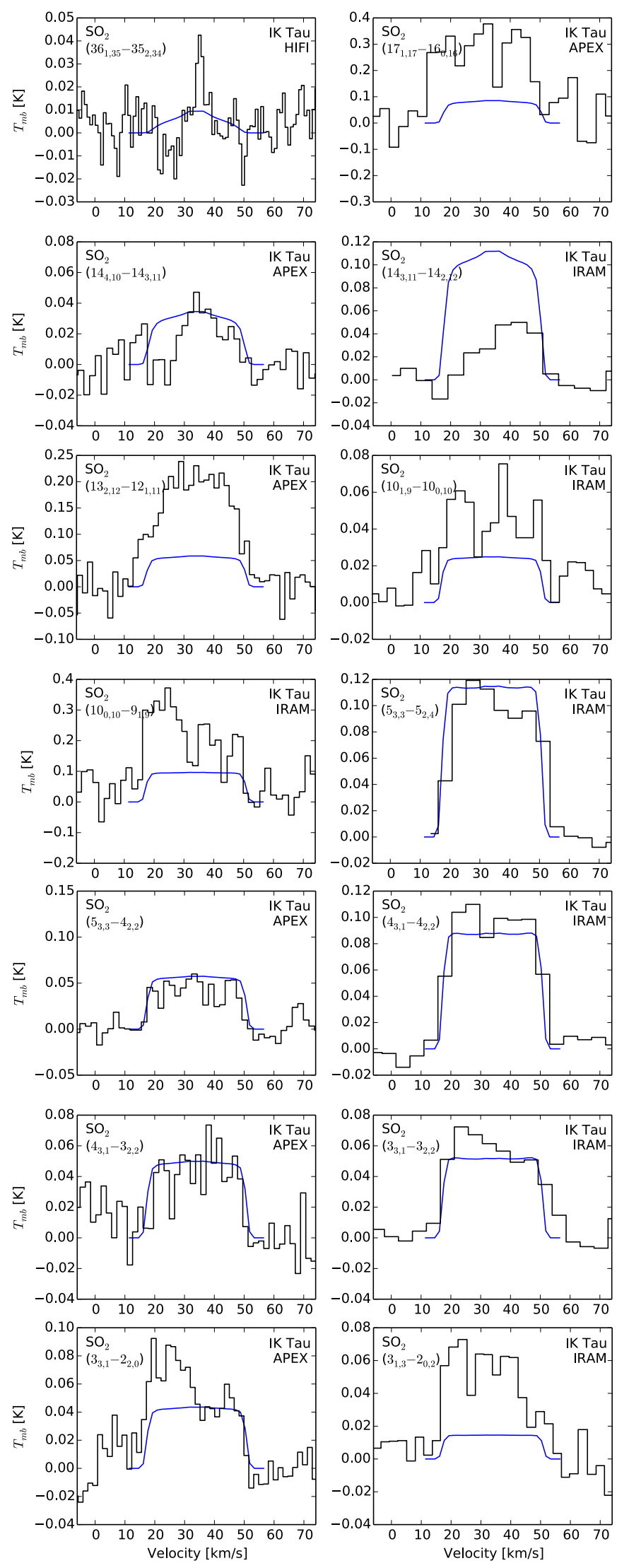

Fig. 13. $\mathrm{SO}_{2}$ model (blue line) and observations (black histograms) of IK Tau. For details on the archival observations, see Table C.3.

a result of unusual structure in the CSE of IK Tau or could be a result of not being able to properly consider overlaps in the
$\mathrm{SO}_{2}$ model. The higher wind velocity would also lead to more overlapping lines overall - including in regions we have not observed - which could have an effect on the overall energy distribution between all molecular energy levels.

Kim et al. (2010) use a combination of Monte-Carlo radiative transfer modelling, to find CSE properties, and LTE formulations, to determine $\mathrm{SO}$ and $\mathrm{SO}_{2}$ abundance for IK Tau. For SO they find fractional abundances in the range 3 to $8 \times 10^{-7}$ and for $\mathrm{SO}_{2}$ their fractional abundances were in the range $4 \times 10^{-6}$ to $1 \times 10^{-5}$. Their results are not accompanied by clear abundance distributions, making them difficult to compare with our results. Nevertheless, their SO result is very close to our peak abundance for $\mathrm{SO}$, while their $\mathrm{SO}_{2}$ result is much higher than we found.

\subsection{3. $\mathrm{R}$ Cas}

As with IK Tau, we find that a model with a centrally peaked Gaussian distribution of SO does not match the observed data. We again run a three-parameter grid to find the best shell-model fit to the data and find $f_{\mathrm{p}}=(6.0 \pm 1.2) \times 10^{-6}, R_{\mathrm{p}}=(3.2 \pm 0.3) \times$ $10^{15} \mathrm{~cm}$, and $R_{\mathrm{w}}=1.0 R_{\mathrm{p}} \mathrm{cm}$ (gridded in steps of $0.2 R_{\mathrm{p}}$ ), with $\chi_{\text {red }}^{2}=3.12$. The resultant model lines are shown in Fig. 14 with the observations. The $\chi^{2}$ plot for SO in R Cas is shown in Fig. 3 and the goodness of fit plot is included in Fig. 5.

As there are only $2 \mathrm{SO}_{2}$ lines observed towards $\mathrm{R}$ Cas, we are only able to find an approximate model for $\mathrm{SO}_{2}$. As with IK Tau, a shell-like model based on the $\mathrm{R}$ Cas $\mathrm{SO}$ results does not fit the $\mathrm{SO}_{2}$ observations. Our best model has $f_{\mathrm{p}}=7 \times 10^{-6}$ (best within steps of $1 \times 10^{-6}$ ) and $R_{e}=6 \times 10^{15} \mathrm{~cm}$ (best within steps of $1 \times 10^{15} \mathrm{~cm}$ ). In Fig. 15 we plot the HIFI detection with our model. An abundance plot for $\mathrm{SO}, \mathrm{SO}_{2}, \mathrm{CO}$ and $\mathrm{H}_{2} \mathrm{O}$ towards $\mathrm{R}$ Cas is shown in Fig. 8.

We note that the HIFI detection in Fig. 15 has a central narrow peak, much narrower than the gas expansion velocity, This skews the overall integrated line intensity somewhat. Interestingly, IK Tau has a similar narrow peak in the same transition line (see Fig. 13), also at approximately the stellar velocity. R Dor does not have such a peak and W Hya may have one which is significantly less bright with respect to the rest of the emission line. The cause of this feature is unclear.

\subsubsection{TX Cam}

In the case of SO towards TX Cam, we do not have sufficient constraints to run a full grid and perform a $\chi^{2}$ analysis as we did for the other stars. Instead we aim to fit the archival lines and find the largest $R_{\mathrm{w}}$ allowed by the HIFI non-detections. The best model with these assumptions has $f_{\mathrm{p}}=1.7 \times 10^{-6}$, $R_{\mathrm{p}}=1.4 \times 10^{16} \mathrm{~cm}$ and $R_{\mathrm{w}}=1.6 R_{\mathrm{p}}$. We plot the detected lines with our model in Fig. 16. The goodness of fit, represented by the ratio between model integrated intensities and observed integrated intensities, is plotted in Fig. 5. We stress that the dearth of observational results leaves our model poorly constrained and this is just one possible model that fits the available data. We can, however, rule out a centrally peaked model, as in that case we would expect the HIFI lines to have been detected, given the constraints on the fit from the archival lines.

There were no $\mathrm{SO}_{2}$ lines detected towards TX Cam. 
T. Danilovich et al.: Sulphur molecules in the circumstellar envelopes of M-type AGB stars
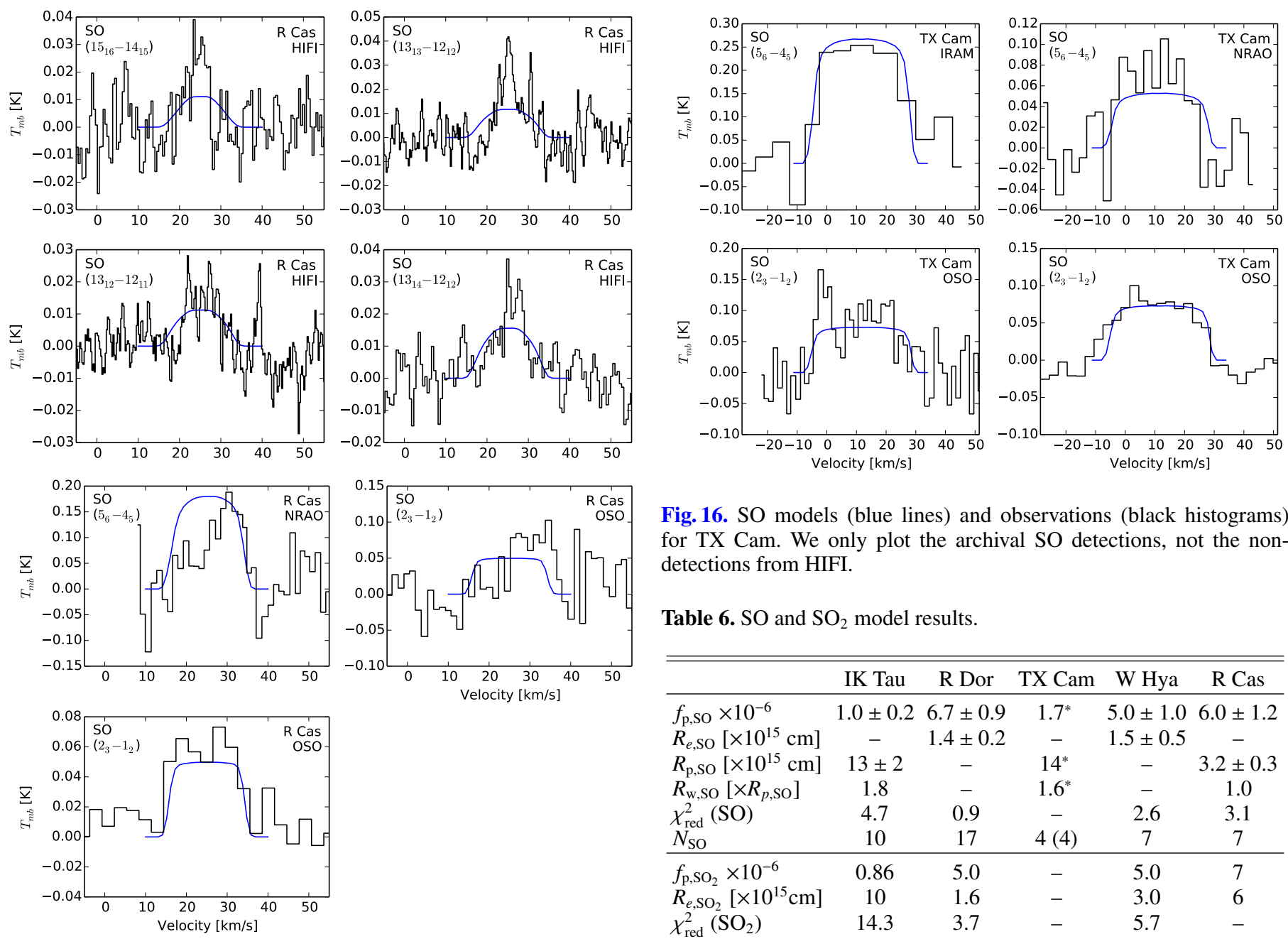

Fig. 16. SO models (blue lines) and observations (black histograms) for TX Cam. We only plot the archival SO detections, not the nondetections from HIFI.

Table 6. $\mathrm{SO}$ and $\mathrm{SO}_{2}$ model results.

\begin{tabular}{lccccc}
\hline \hline & IK Tau & R Dor & TX Cam & W Hya & R Cas \\
\hline$f_{\mathrm{p}, \mathrm{SO}} \times 10^{-6}$ & $1.0 \pm 0.2$ & $6.7 \pm 0.9$ & $1.7^{*}$ & $5.0 \pm 1.0$ & $6.0 \pm 1.2$ \\
$R_{e, \mathrm{SO}}\left[\times 10^{15} \mathrm{~cm}\right]$ & - & $1.4 \pm 0.2$ & - & $1.5 \pm 0.5$ & - \\
$R_{\mathrm{p}, \mathrm{SO}}\left[\times 10^{15} \mathrm{~cm}\right]$ & $13 \pm 2$ & - & $14^{*}$ & - & $3.2 \pm 0.3$ \\
$R_{\mathrm{w}, \mathrm{SO}}\left[\times R_{p, \mathrm{SO}}\right]$ & 1.8 & - & $1.6^{*}$ & - & 1.0 \\
$\chi_{\mathrm{red}}^{2}(\mathrm{SO})$ & 4.7 & 0.9 & - & 2.6 & 3.1 \\
$N_{\mathrm{SO}}$ & 10 & 17 & $4(4)$ & 7 & 7 \\
\hline$f_{\mathrm{p}, \mathrm{SO}} \times 10^{-6}$ & 0.86 & 5.0 & - & 5.0 & 7 \\
$R_{e, \mathrm{SO}_{2}}\left[\times 10^{15} \mathrm{~cm}\right]$ & 10 & 1.6 & - & 3.0 & 6 \\
$\chi_{\mathrm{red}}^{2}\left(\mathrm{SO}_{2}\right)$ & 14.3 & 3.7 & - & 5.7 & - \\
$N_{\mathrm{SO}_{2}}$ & 14 & 98 & 0 & 5 & 2 \\
\hline
\end{tabular}

Fig. 14. SO models (blue lines) and observations (black histograms) for R Cas.

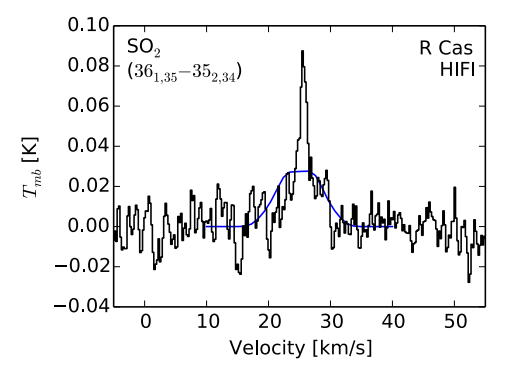

Fig. 15. $\mathrm{SO}_{2}$ model (blue line) and observation (black histogram) for $\mathrm{R}$ Cas.

\section{Discussion}

\subsection{SO distribution}

Our results for circumstellar SO are summarised in Table 6. In Fig. 17 we plot the circumstellar SO abundance profiles of the stars we modelled. We also show the radial range probed by the available observational data for each line with a thicker line. These ranges were found by considering the brightness distributions for each emission line and the radii at which these fall to half of their maximum values. It is interesting to note that for R Cas, IK Tau, and TX Cam, the three stars with shell-like SO distributions, the location of the peak is found progressively

Notes. $N$ is the number of lines used to constrain our models. The uncertainties are for the $90 \%$ confidence level. The number in brackets for $N$ is the number of upper limits used in addition to the detected lines. Values marked with a ${ }^{(*)}$ indicate an upper-limit model.

further out with increasing mass-loss rate. The two low massloss rate stars, however, both seem to have centrally peaked SO distributions, which could be interpreted as shells with peaks close to the star, especially if the peaks are near or within our inner radii. Looking at the three stars with shell-like SO distributions, there also seems to be a trend of decreasing SO abundance with increasing mass-loss rate (or with the radius of peak SO abundance).

In Fig. 18 we plot the peak positions against the wind density, $\dot{M} / v_{\infty}$, and fit a power law to the three higher mass-loss rate stars (R Cas, TX Cam and IK Tau). The results for these stars are well fit by a power law

$R_{\mathrm{p}} \propto\left(\frac{\dot{M}}{v_{\infty}}\right)^{\alpha_{R}}$

with $\alpha_{R}=1.15 \pm 0.24$. We extend the power law to predict the peak positions for R Dor and W Hya, were they also to fit this trend. This predicts the peak in SO abundance for R Dor to lie at $1.0 \times 10^{15} \mathrm{~cm}$, which is close to the $R_{e}$ we found, and for W Hya the predicted SO peak lies at $4.4 \times 10^{14} \mathrm{~cm}$, about three times smaller than the $e$-folding radius. Running models with shelllike distributions at the predicted peaks, we found they could 


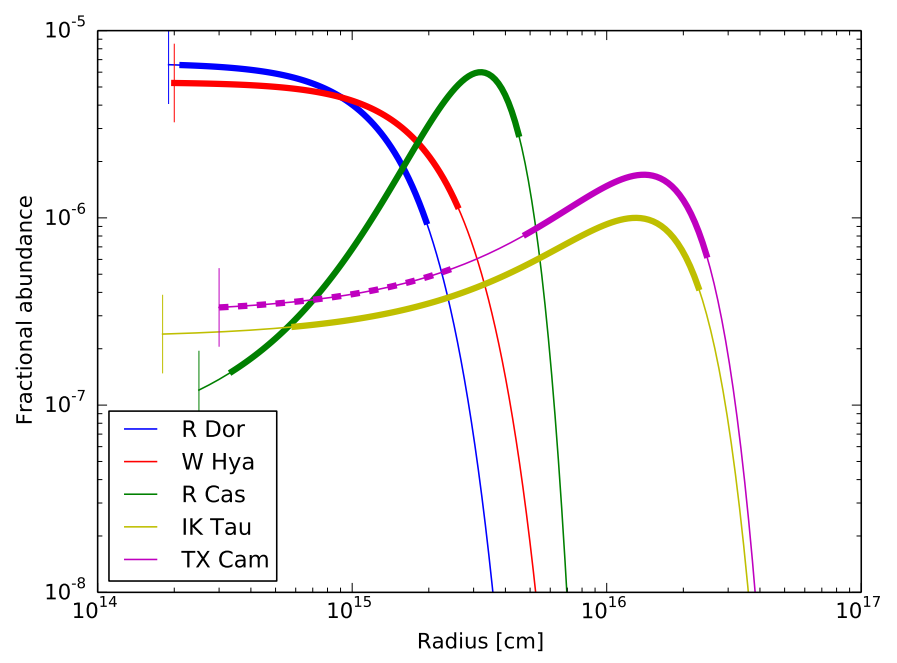

Fig. 17. SO abundance distributions for all stars modelled. The vertical lines represent the dust condensation radii, where our models stop. The thicker sections of the curves represent the area probed by our observations and for TX Cam the thick dashed line is the area probed by the upper limits imposed by the HIFI non-detections.

not provide as good a fit for either R Dor or W Hya as the starcentred Gaussian models. In general, the best shell models had at least twice the $\chi^{2}$ values of the best star-centred Gaussian models. Given the region probed by our observations as shown in Fig. 17, it is not surprising that changing the inner abundance would have an effect on the model fit.

We performed a similar fit for the peak abundance values against density for the three highest mass-loss rate stars. The results for these stars are well fit by a power law

$f_{\mathrm{p}} \propto\left(\frac{\dot{M}}{v_{\infty}}\right)^{\alpha_{\mathrm{f}}}$

with $\alpha_{\mathrm{f}}=-1.29 \pm 0.17$, Fig. 18 . Doing a similar extrapolation to predict the peak abundance values for R Dor and W Hya based on the power law, we find fractional abundance predictions of $2.2 \times 10^{-5}$ for R Dor and $5.8 \times 10^{-5}$ for W Hya. Both of these are higher than the values we find from our modelling and in the case of W Hya this represents more sulphur than should be available, i.e. it exceeds the solar and ISM abundances (see below). Because the SO abundance cannot increase with decreased mass-loss rate indefinitely, there must be a maximum SO abundance set by the abundance of sulphur.

The shell-like distributions of SO for the three stars with the highest mass-loss rates means that circumstellar chemistry, most likely related to photodissociation, must play an important role, in these cases. It is therefore interesting to compare with results on photodissociation for other species. $\mathrm{H}_{2} \mathrm{O}$ is particularly interesting here, since, as will be discussed below, $\mathrm{SO}$ (and also $\mathrm{SO}_{2}$ ) may owe its origin to the presence of circumstellar $\mathrm{OH}$, which in turn is a photodissociation product of $\mathrm{H}_{2} \mathrm{O}$. Netzer \& Knapp (1987) predict a peak $\mathrm{OH}$ radius that scales with both mass-loss rate and expansion velocity. Their formulation has been used by Maercker et al. (2008, 2009), Schöier et al. (2011), Danilovich et al. (2014) and others to define the $e$ folding radius of $\mathrm{H}_{2} \mathrm{O}$, since $\mathrm{OH}$ is a photodissociation product of $\mathrm{H}_{2} \mathrm{O}$ and peaks in abundance where $\mathrm{H}_{2} \mathrm{O}$ drops off. In Fig. 19 we plot our SO peak abundance radii or $e$-folding radii (as relevant) against the $\mathrm{H}_{2} \mathrm{O} e$-folding radii of the same stars found by Maercker et al. (in prep.) and Khouri et al. (2014b, for W Hya).
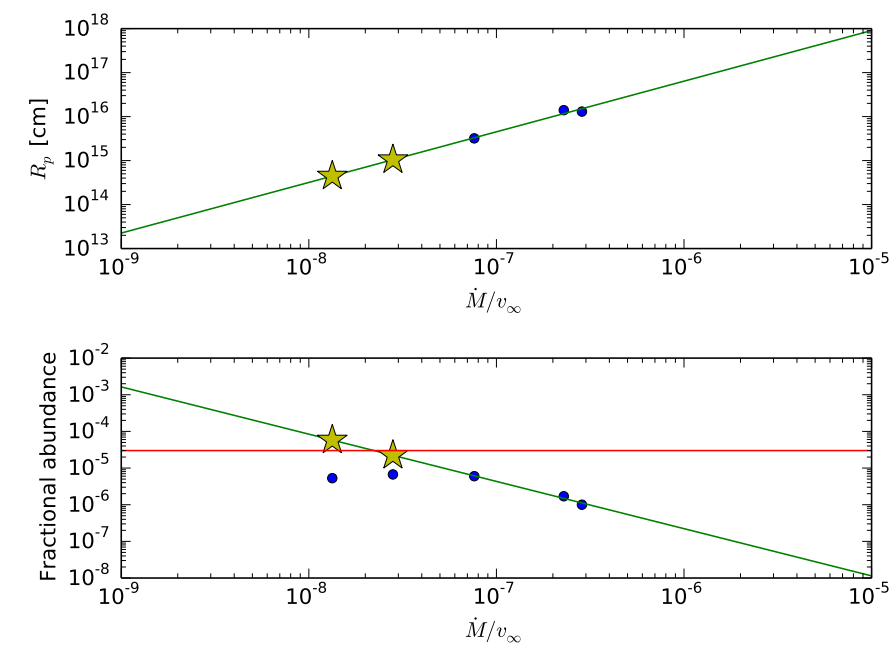

Fig. 18. Trends in SO peak radius (top) and fractional abundance (bottom) against the circumstellar density measure, $\dot{M} / v_{\infty}$. The green lines are the trends fitted to the three higher mass-loss rate stars (R Cas, TX Cam and IK Tau), while the yellow stars are the predicted locations of the lower mass-loss rate stars (R Dor and W Hya) based on the trend. In the fractional abundance plot, the red line shows the hard limit for SO abundance based on solar S abundance and the blue points in line with the yellow stars represent the real abundance values for R Dor and W Hya.

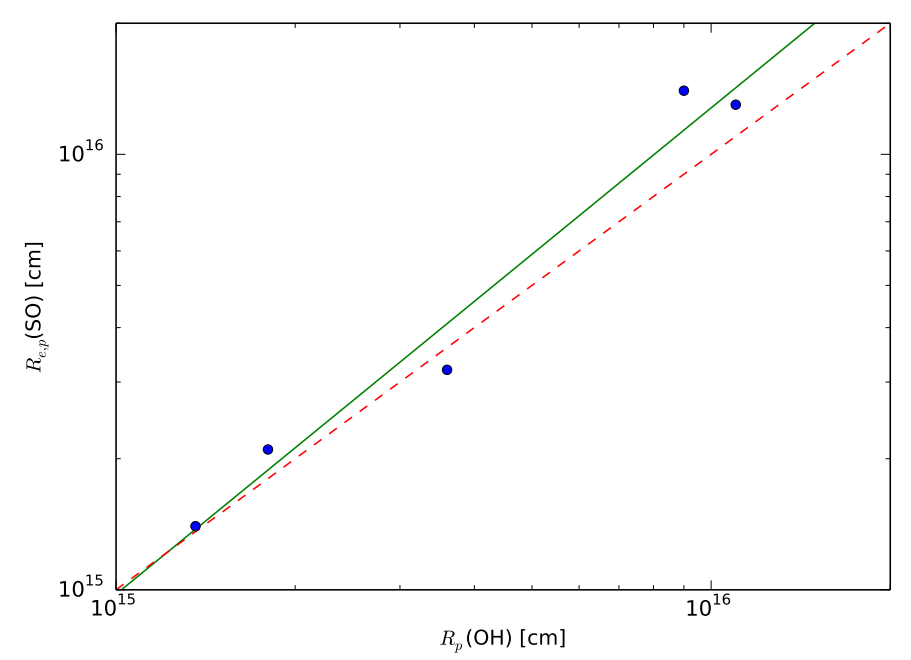

Fig. 19. Peak abundance radii of SO (for R Cas, TX Cam, and IK Tau) and $e$-folding radii of SO (for R Dor and W Hya), plotted against the $e$-folding radii of $\mathrm{H}_{2} \mathrm{O}$ found by Maercker et al. (in prep.) and Khouri et al. (2014b, for W Hya). The solid green line is the best fit to the data and the dashed red line traces a 1:1 relationship.

We find a strong correlation, which is close to being 1:1. The chemistry of SO will be discussed in Sect. 4.3.

\section{2. $\mathrm{SO}_{2}$ distribution}

Our results for circumstellar $\mathrm{SO}_{2}$ are summarised in Table 6 . For R Dor and W Hya we find circumstellar $\mathrm{SO}_{2}$ distributions of similar size and abundance to the circumstellar SO distributions. Our results for the higher mass-loss rate stars, however, are less clear. For both IK Tau and R Cas, the best models are centrally-peaked Gaussian distributions of $\mathrm{SO}_{2}$, rather than the 
shell-like models we found for SO. For IK Tau, we were unable to constrain the $e$-folding radius better than by a factor of 2 and for R Cas we only had two observations, but in both cases shell models similar to the corresponding SO models are ruled out. Our $\mathrm{SO}_{2}$ results for IK Tau and $\mathrm{R}$ Cas suggest that $\mathrm{SO}_{2}$ is formed in the inner regions irrespective of the mass-loss rate. It appears that $\mathrm{SO}_{2}$ is formed more favourably than $\mathrm{SO}$ in the inner regions. There does not appear to be a strong correlation between $e$-folding radius and mass-loss rates or $\mathrm{H}_{2} \mathrm{O} e$-folding radii, as we found for $\mathrm{SO}$, but this might become clearer if we add more $\mathrm{SO}_{2}$ observations to our models, especially for $\mathrm{R}$ Cas and $\mathrm{W}$ Hya. We emphasise that our $\mathrm{SO}_{2}$ results for the higher mass-loss rate stars, R Cas and IK Tau, are particularly uncertain.

\subsection{Comparisons with chemical models}

There exist two studies of the abundances of $\mathrm{SO}$ and $\mathrm{SO}_{2}$ in the extended atmospheres of AGB stars (Cherchneff 2006; Gobrecht et al. 2016). In both cases the effects of shock-induced chemistry, due to pulsational motion, are included. Cherchneff (2006) has chosen TX Cam as the representative star, and the modelling covers the region from 1 to 5 stellar radii, $R_{*}$, which means that the outer reach of their model is approximately an order of magnitude smaller than our inner radii. For their model star with $\mathrm{C} / \mathrm{O}=0.75$, they find an $\mathrm{SO}$ abundance at $5 R_{*}$ of $3 \times 10^{-7}$, and an $\mathrm{SO}_{2}$ abundance several orders of magnitude lower than this. Gobrecht et al. (2016) use IK Tau as the example, and extend the calculations to 10 stellar radii (the outer radius of their model then approximately meets the inner radius used by us). Their model focuses on the shock chemistry in this region, much of which varies with the pulsation phase. Their abundances of $\mathrm{SO}$ and $\mathrm{SO}_{2}$ are much lower than we observe, at $\sim 10^{-8}$ and $\sim 2 \times 10^{-9}$, respectively. This means that the predicted SO and $\mathrm{SO}_{2}$ abundances close to the star, at least for these higher massloss rate stars, are substantially lower than we derive for our sample stars.

The shell-like SO distributions for the higher mass-loss rate stars suggest a circumstellar origin. Willacy \& Millar (1997) describe circumstellar chemical models of four M-type AGB stars, including R Dor, TX Cam, and IK Tau. Their models differ from ours in terms of CSE parameters, for example taking the inner radius to be $2 \times 10^{15} \mathrm{~cm}$, about an order of magnitude larger than our inner radii. They assume that all the sulphur is carried by $\mathrm{H}_{2} \mathrm{~S}$ that is eventually photodissociated. $\mathrm{SO}$ is subsequently formed through the following reactions

$\mathrm{S}+\mathrm{OH} \rightarrow \mathrm{SO}+\mathrm{H}$

$\mathrm{SH}+\mathrm{O} \rightarrow \mathrm{SO}+\mathrm{H}$

which are favoured depending on the availability of $\mathrm{OH}$ and $\mathrm{SH}$, respectively, and with Eq. 8 dominating at the lower gas temperatures in the CSE. Following this, SO can be destroyed through

$\mathrm{SO}+\mathrm{OH} \longleftrightarrow \mathrm{SO}_{2}+\mathrm{H}$

and hence form $\mathrm{SO}_{2}$. Unfortunately, they only visualise their model results for TX Cam, the least well-constrained star of our sample. Nevertheless, the location of the peak of the SO distribution that we find for TX Cam agrees quite well with their predicted peak location. Our peak abundance is about $50 \%$ higher than theirs, but this must be considered to be within the errors. Their $\mathrm{SO}_{2}$ distribution for TX Cam peaks at roughly the same radius as $\mathrm{SO}$, but with a peak abundance about an order of magnitude lower than that of $\mathrm{SO}$ (as we do not have any $\mathrm{SO}_{2}$ detections for TX Cam, we cannot compare this directly). The molecular column densities they list for R Dor, IK Tau, and TX Cam are, in general, a few orders of magnitude lower than those predicted by our models.

In conclusion, our SO results for the outer CSE are reasonably consistent with the results of Willacy \& Millar (1997) for the higher mass-loss rate objects, although they do not predict a peak abundance that decreases with mass-loss rate. Thus, an origin through $\mathrm{OH}$ is likely, a result that is further strengthened by the correlation between $\mathrm{SO}$ and $\mathrm{H}_{2} \mathrm{O}$ sizes that we found. However, we note that neither Cherchneff (2006) nor Gobrecht et al. (2016) predict high abundances of $\mathrm{H}_{2} \mathrm{~S}$ in the upper atmosphere. For the lower mass-loss rate objects, where the SO abundance is high close to the star, the models of Cherchneff (2006) and Gobrecht et al. (2016) fail by more than two orders of magnitude to reproduce our estimated abundances. They even fail to reproduce the inner $\mathrm{SO}$ abundances for the higher mass-loss rate stars.

In the case of $\mathrm{SO}_{2}$ we find no evidence for a photo-induced circumstellar origin along the Willacy \& Millar (1997) model for any of our objects. Once again, we caution that the results for $\mathrm{R}$ Cas and IK Tau are uncertain. The $\mathrm{SO}_{2}$ abundances that we estimate for R Dor and W Hya are an order of magnitude higher than those predicted by Cherchneff (2006) and Gobrecht et al. (2016).

\subsection{Sulphur chemistry: can we account for all the sulphur?}

AGB stars and their progenitors do not produce $S$ via nucleosynthesis. As such, the quantity of sulphur available to form molecules in the CSE of an AGB star is fixed and not dependent on the stage of evolution or mass of the star in question. Rudolph et al. (2006) find an $\mathrm{S} / \mathrm{H}$ ratio in the ISM of $\sim 10^{-5}$ in the solar neighbourhood and Lodders (2003) indicate a solar $\mathrm{S} / \mathrm{H}$ abundance of $1.5 \times 10^{-5}$. All stars in our sample are at distances $<400 \mathrm{pc}$ and hence can be assumed to trace a similar $\mathrm{S} / \mathrm{H}$ abundance. In this work we refer to the fractional molecular abundance with respect to $\mathrm{H}_{2}$. Hence, assuming all hydrogen is in the form of $\mathrm{H}_{2}$ in the CSE, we will take the $\mathrm{S} / \mathrm{H}_{2}$ ratio to be $\sim 2$ to $3 \times 10^{-5}$, which represents the maximum total amount of sulphur that should be found in an AGB star.

For R Dor and W Hya we find combined $\mathrm{SO}$ and $\mathrm{SO}_{2}$ abundances of $\sim 1.2 \times 10^{-5}$ and $\sim 1.0 \times 10^{-5}$, respectively. Hence, in these cases most of the sulphur is locked up in $\mathrm{SO}$ and $\mathrm{SO}_{2}$ within the inner regions of the CSE and within the errors. This result is consistent with the non-detections (or low-level emission) for CS and SiS in the APEX spectral scan of R Dor, and no reported detections of these species towards W Hya. In the case of $\mathrm{R} \mathrm{Cas}$, the combined $\mathrm{SO}$ and $\mathrm{SO}_{2}$ abundances in the mid-CSE is $\sim 1.4 \times 10^{-5}$, suggesting that these two species carry all the sulphur, but here the uncertainty on the $\mathrm{SO}_{2}$ abundance is substantial. For the high mass-loss rate object IK Tau, the combined $\mathrm{SO}$ and $\mathrm{SO}_{2}$ abundance is well below that of sulphur.

In general, higher mass-loss rate stars also show definitive detections of other S-bearing molecules. For example, SiS was detected in several carbon and M-type stars by Schöier et al. (2007) and Danilovich et al. (2015). Schöier et al. (2007) reported circumstellar SiS abundances of $4 \times 10^{-7}, 4 \times 10^{-7}$, and $1 \times 10^{-7}$ for R Cas, TX Cam, and IK Tau, respectively, suggesting that $\mathrm{SiS}$ is less abundant than $\mathrm{SO}$ and $\mathrm{SO}_{2}$ by up to an order of magnitude, at least in the outer CSE. It should be noted that Schöier et al. (2007) assume a Gaussian distribution of SiS centred on the star, but find their model fit greatly improved when they include a high-abundance inner component, which could represent the SiS reservoir before depletion through dust 
condensation. Decin et al. (2010a), who model IK Tau in detail, also find evidence of depletion of SiS.

Another S-bearing molecule, CS, has mainly been detected in carbon stars rather than M-type stars. CS has been detected and modelled in IK Tau by Kim et al. (2010) and Decin et al. (2010a), detected in TX Cam and IK Tau by Bujarrabal et al. (1994, with non-detections in R Cas and W Hya) and Lindqvist et al. (1988, with a non-detection in R Cas). Derived CS abundances for M-type stars have generally been low, in the range $\sim 10^{-8}$ to $\sim 5 \times 10^{-8}$ (see Bujarrabal et al. 1994; Decin et al. 2010a, for examples).

$\mathrm{H}_{2} \mathrm{~S}$ is considered as a parent species of sulphur in the chemical modelling of Willacy \& Millar (1997). However, $\mathrm{H}_{2} \mathrm{~S}$ has not been widely detected in AGB stars other than in OH/IR stars. For example, in the HIFISTARS project $\mathrm{H}_{2} \mathrm{~S}$ was only detected in AFGL 5379 (Justtanont et al. 2012), despite being in the observed range for all stars except TX Cam, while Justtanont et al. (2015) detected $\mathrm{H}_{2} \mathrm{~S}$ in all OH/IR stars observed with SPIRE and some observed with PACS. In a study of 25 stars, Ukita \& Morris (1983) detected $\mathrm{H}_{2} \mathrm{~S}$ only in $\mathrm{OH} 231.8+4.2$ aka the Rotten Egg Nebula. Omont et al. (1993) detected $\mathrm{H}_{2} \mathrm{~S}$ in several high massloss rate stars, including several $\mathrm{OH} / \mathrm{IR}$ stars. Of the stars we modelled, Ukita \& Morris (1983) did not detect $\mathrm{H}_{2} \mathrm{~S}$ in W Hya, $\mathrm{R}$ Cas, and TX Cam, but it was detected in IK Tau by Omont et al. (1993) and De Beck et al. (in prep.). This suggests that $\mathrm{H}_{2} \mathrm{~S}$ may require high densities to form, or may be able to survive longer in the CSEs of high mass-loss rate stars, or that the excitation conditions are such that the emission is only bright enough in the very high mass-loss rate stars to be detectable. We note that Gobrecht et al. (2016) predict a fairly rapid decline of $\mathrm{H}_{2} \mathrm{~S}$ inside of the dust condensation radius (which is where our models start) even for IK Tau, which is a relatively high massloss rate object.

To check what the lack of detections predicts in terms of $\mathrm{H}_{2} \mathrm{~S}$ abundances, we run radiative transfer models for $\mathrm{R}$ Dor and IK Tau to find upper limits for the $\mathrm{H}_{2} \mathrm{~S}$ abundances based on the non-detections in HIFI, the non-detection in APEX for R Dor, and the SMA detection in IK Tau by De Beck et al. (in prep.). We used the ortho- $\mathrm{H}_{2} \mathrm{~S}$ molecular data file available on LAMDA ${ }^{5}$ (Schöier et al. 2005) which includes the lowest 45 rotational energy levels, 139 radiative transitions with frequencies taken from $\mathrm{JPL}^{6}$ and 990 collisional transitions taken from Dubernet et al. (2009) for temperatures from 5-1500 K. For IK Tau, using the detection from De Beck et al. (in prep.) and the HIFI upper limit to also constrain the envelope size, we find a small envelope with $R_{e} \simeq 4 \times 10^{14} \mathrm{~cm}$ and $f_{\mathrm{p}} \simeq 4 \times 10^{-6}$. This is consistent with a rapid destruction of $\mathrm{H}_{2} \mathrm{~S}$. For R Dor, using both non-detections and assuming the $R_{e}$ we find for $\mathrm{SO}$, we find an upper limit on the abundance of $f_{\mathrm{p}} \lesssim 2.5 \times 10^{-7}$. If we instead use the $\mathrm{H}_{2} \mathrm{~S}$ envelope size found for IK Tau, the abundance upper limit increases slightly to $f_{\mathrm{p}} \lesssim 6 \times 10^{-7}$. In any case, these results limit the possibility that $\mathrm{H}_{2} \mathrm{~S}$ is a significant $\mathrm{S}$-carrier in the inner CSE, certainly for the low mass-loss rate objects.

None of the molecules discussed thus far have been found in sufficient quantities towards higher mass-loss rate AGB stars to account for the full amount of expected sulphur. It is possible that the remaining sulphur is locked up in dust or left as atomic $\mathrm{S}$ or locked up in molecules that are difficult to detect for various reasons, such as the spectral region they are most likely to emit in, as is the case with HS. Both Cherchneff (2006) and

\footnotetext{
5 The Leiden Atomic and Molecular Database, found at http://home.strw.leidenuniv.nl/ moldata/

${ }^{6}$ http://spec.jpl.nasa.gov/
}

Willacy \& Millar (1997) predict a rapid decline of HS with radius as it is consumed by various chemical processes (although we note that the two studies make predictions for different regions around the star). The only detection of HS in the literature is through ro-vibrational lines identified by Yamamura et al. (2000) towards R And (an S-type AGB star). They estimate a molecular abundance of $\mathrm{HS} / \mathrm{H} \sim 1 \times 10^{-7}$, which is well below the sulphur limit. There have been no other detections of circumstellar HS, although it has been detected in the ISM (see e.g. Neufeld et al. 2015).

To fully study the issue of sulphur in the CSEs of AGB stars of different mass-loss rates, a more thorough investigation including more molecular species - such as $\mathrm{SiS}, \mathrm{CS}$, and $\mathrm{H}_{2} \mathrm{~S}$ in addition to $\mathrm{SO}$ and $\mathrm{SO}_{2}$ - across a larger sample of stars is needed.

\section{Conclusions}

We present new APEX observations of a very large number of $\mathrm{SO}$ and $\mathrm{SO}_{2}$ lines towards the low mass-loss rate M-type AGB star R Dor. Combining these data with higher-frequency observations from Herschel/HIFI, we compute comprehensive radiative transfer models to determine the molecular abundances and distributions of the two molecules. For R Dor we find a Gaussian abundance distribution centred on the star, with a peak SO fractional abundance of $(6.7 \pm 0.9) \times 10^{-6}$ and $e$-folding radius of $(1.4 \pm 0.2) \times 10^{15} \mathrm{~cm}$, and an $\mathrm{SO}_{2}$ fractional abundance of $5.0 \times 10^{-6}$ and $e$-folding radius of $1.6 \times 10^{15} \mathrm{~cm}$. Our ${ }^{34} \mathrm{SO}$ model assumes the same $e$-folding radius as for ${ }^{32} \mathrm{SO}$ and we find an abundance of $(3.1 \pm 0.8) \times 10^{-7}$. This gives an ${ }^{32} \mathrm{SO} /{ }^{34} \mathrm{SO}$ ratio of $21.6 \pm 8.5$, which is in agreement with previous results from other nearby stars.

We also model SO in four other M-type AGB stars that were observed as part of HIFISTARS: IK Tau, TX Cam, W Hya, and $\mathrm{R}$ Cas. For TX Cam for we are only able to provide an upper limit model since there are no SO lines detected with HIFI. Of these four stars only W Hya has a similar SO distribution to $\mathrm{R}$ Dor. The other three stars, all of which have higher mass-loss rates, are best fit with shell-like abundance distributions. We find that the radial position of the peak of the distributions increases with mass-loss rate, while the peak abundances decrease. The location of the peaks of the SO distributions correlates with the photodissociation of $\mathrm{H}_{2} \mathrm{O}$ into $\mathrm{OH}$ (itself partly dependent on mass-loss rate), suggesting that the production of SO depends on the availability of $\mathrm{OH}$ to participate in the formation process.

We are only able to model $\mathrm{SO}_{2}$ in an additional three stars, IK Tau, W Hya, and R Cas, owing to the dearth of detections towards TX Cam. For W Hya we find an $\mathrm{SO}_{2}$ distribution similar to $\mathrm{SO}$ in abundance and envelope size. We have some difficulty fitting an $\mathrm{SO}_{2}$ model to observations for IK Tau and ultimately find an uncertain model which differs in shape from the SO distribution. For R Cas the $\mathrm{SO}_{2}$ model is also very uncertain because there are only two detected lines.

Overall, the circumstellar $\mathrm{SO}$ and $\mathrm{SO}_{2}$ abundances are much higher than predicted by chemical models of the extended stellar atmosphere. These two species may also account for all the available sulphur in the lower mass-loss rate stars. The S-bearing parent molecule appears not to be $\mathrm{H}_{2} \mathrm{~S}$. The $\mathrm{SO}_{2}$ models for the higher mass-loss rate stars are less conclusive, but suggest an origin close to the star for this species. This is not consistent with present chemical models. The combined circumstellar SO and $\mathrm{SO}_{2}$ abundances are significantly lower than that of sulphur for these higher mass-loss rate objects. 
To better constrain the behaviour of sulphur we need more observations of $\mathrm{SO}$ and $\mathrm{SO}_{2}$, as well as other S-bearing species. Observations of a larger sample of stars will also allow us to confirm the trends we see in the SO abundance distributions.

Acknowledgements. T.D. and K.J. acknowledge funding from the Swedish National Space Board. HO acknowledges financial support from the Swedish Research Council. This publication is based on data acquired with the Atacama Pathfinder Experiment (APEX). APEX is a collaboration between the MaxPlanck-Institut für Radioastronomie, the European Southern Observatory, and the Onsala Space Observatory. HIFI has been designed and built by a consortium of institutes and university departments from across Europe, Canada and the United States under the leadership of SRON Netherlands Institute for Space Research, Groningen, The Netherlands and with major contributions from Germany, France and the US. Consortium members are: Canada: CSA, U.Waterloo; France: CESR, LAB, LERMA, IRAM; Germany: KOSMA, MPIfR, MPS; Ireland, NUI Maynooth; Italy: ASI, IFSI-INAF, Osservatorio Astrofisico di Arcetri-INAF; Netherlands: SRON, TUD; Poland: CAMK, CBK; Spain: Observatorio Astronómico Nacional (IGN), Centro de Astrobiología (CSIC-INTA). Sweden: Chalmers University of Technology - MC2, RSS \& GARD; Onsala Space Observatory; Swedish National Space Board, Stockholm University - Stockholm Observatory; Switzerland: ETH Zurich, FHNW; USA: Caltech, JPL, NHSC.

\section{References}

Adande, G. R., Edwards, J. L., \& Ziurys, L. M. 2013, ApJ, 778, 22 Bujarrabal, V., Fuente, A., \& Omont, A. 1994, A\&A, 285, 247 Burkholder, J. B., Lovejoy, E. R., Hammer, P. D., Howard, C. J., \& Mizushima, M. 1987, J. Mol. Spectr., 124, 379

Cameron, A. G. W. 1973, Space Sci. Rev., 15, 121

Cami, J., Yamamura, I., de Jong, T., et al. 1999, in The Universe as Seen by ISO, eds. P. Cox, \& M. Kessler, ESA SP, 427, 281

Cernicharo, J., Spielfiedel, A., Balança, C., et al. 2011, A\&A, 531, A103

Cherchneff, I. 2006, A\&A, 456, 1001

Danilovich, T., Bergman, P., Justtanont, K., et al. 2014, A\&A, 569, A76 Danilovich, T., Teyssier, D., Justtanont, K., et al. 2015, A\&A, 581, A60 de Graauw, T., Helmich, F. P., Phillips, T. G., et al. 2010, A\&A, 518, L6 Decin, L., De Beck, E., Brünken, S., et al. 2010a, A\&A, 516, A69 Decin, L., Justtanont, K., De Beck, E., et al. 2010b, A\&A, 521, L4 Dubernet, M.-L., Daniel, F., Grosjean, A., \& Lin, C. Y. 2009, A\&A, 497, 911 Gobrecht, D., Cherchneff, I., Sarangi, A., Plane, J. M. C., \& Bromley, S. T. 2016, A\&A, 585, A6
Gong, Y., Henkel, C., Spezzano, S., et al. 2015, A\&A, 574, A56

Green, S. 1995, ApJS, 100, 213

Guilloteau, S., Lucas, R., Omont, A., \& Nguyen-Q-Rieu. 1986, A\&A, 165, L1

Justtanont, K., Khouri, T., Maercker, M., et al. 2012, A\&A, 537, A144

Justtanont, K., Barlow, M. J., Blommaert, J., et al. 2015, A\&A, 578, A115

Kahane, C., Gomez-Gonzalez, J., Cernicharo, J., \& Guelin, M. 1988, A\&A, 190, 167

Khouri, T., de Koter, A., Decin, L., et al. 2014a, A\&A, 561, A5

Khouri, T., de Koter, A., Decin, L., et al. 2014b, A\&A, 570, A67

Kim, H., Wyrowski, F., Menten, K. M., \& Decin, L. 2010, A\&A, 516, A68

Lindqvist, M., Nyman, L.-A., Olofsson, H., \& Winnberg, A. 1988, A\&A, 205, L15

Lique, F., Spielfiedel, A., \& Cernicharo, J. 2006, A\&A, 451, 1125

Lodders, K. 2003, ApJ, 591, 1220

Maercker, M., Schöier, F. L., Olofsson, H., Bergman, P., \& Ramstedt, S. 2008, A\&A, 479, 779

Maercker, M., Schöier, F. L., Olofsson, H., et al. 2009, A\&A, 494, 243

Müller, H. S. P., Thorwirth, S., Roth, D. A., \& Winnewisser, G. 2001, A\&A, 370, L49

Müller, H. S. P., Schlöder, F., Stutzki, J., \& Winnewisser, G. 2005, J. Mol. Struct., 742,215

Netzer, N., \& Knapp, G. R. 1987, ApJ, 323, 734

Neufeld, D. A., Godard, B., Gerin, M., et al. 2015, A\&A, 577, A49

Olofsson, H., Lindqvist, M., Nyman, L.-A., \& Winnberg, A. 1998, A\&A, 329, 1059

Omont, A., Lucas, R., Morris, M., \& Guilloteau, S. 1993, A\&A, 267, 490

Ott, S. 2010, in Astronomical Data Analysis Software and Systems XIX, eds.

Y. Mizumoto, K.-I. Morita, \& M. Ohishi, ASP Conf. Ser., 434, 139

Peterson, K. A., \& Woods, R. C. 1990, J. Chem. Phys., 93, 1876

Ramstedt, S., Mohamed, S., Vlemmings, W. H. T., et al. 2014, A\&A, 570, L14

Rothman, L. S., Gordon, I. E., Babikov, Y., et al. 2013, J. Quant. Spectr. Rad. Transf., 130, 4

Rudolph, A. L., Fich, M., Bell, G. R., et al. 2006, ApJS, 162, 346

Sahai, R., \& Wannier, P. G. 1992, ApJ, 394, 320

Schöier, F. L., van der Tak, F. F. S., van Dishoeck, E. F., \& Black, J. H. 2005, A\&A, 432, 369

Schöier, F. L., Bast, J., Olofsson, H., \& Lindqvist, M. 2007, A\&A, 473, 871

Schöier, F. L., Maercker, M., Justtanont, K., et al. 2011, A\&A, 530, A83

Ukita, N., \& Morris, M. 1983, A\&A, 121, 15

Vassilev, V., Meledin, D., Lapkin, I., et al. 2008, A\&A, 490, 1157

Vlemmings, W. H. T., Humphreys, E. M. L., \& Franco-Hernández, R. 2011, ApJ, 728,149

Willacy, K., \& Millar, T. J. 1997, A\&A, 324, 237

Yamamura, I., de Jong, T., Onaka, T., Cami, J., \& Waters, L. B. F. M. 1999, A\&A, 341, L9

Yamamura, I., Kawaguchi, K., \& Ridgway, S. T. 2000, ApJ, 528, L33 


\section{Appendix A: R Dor plots}

Our best fit model lines for $\mathrm{SO}_{2}$ in $\mathrm{R}$ Dor are plotted along with the corresponding observations in Fig. A.1. For more details see Sect. 3.2.2.

The tentative detections of the isotopologues ${ }^{34} \mathrm{SO}_{2}$ and $\mathrm{SO}^{18} \mathrm{O}$ from the APEX survey towards R Dor are plotted in Fig. A.2.
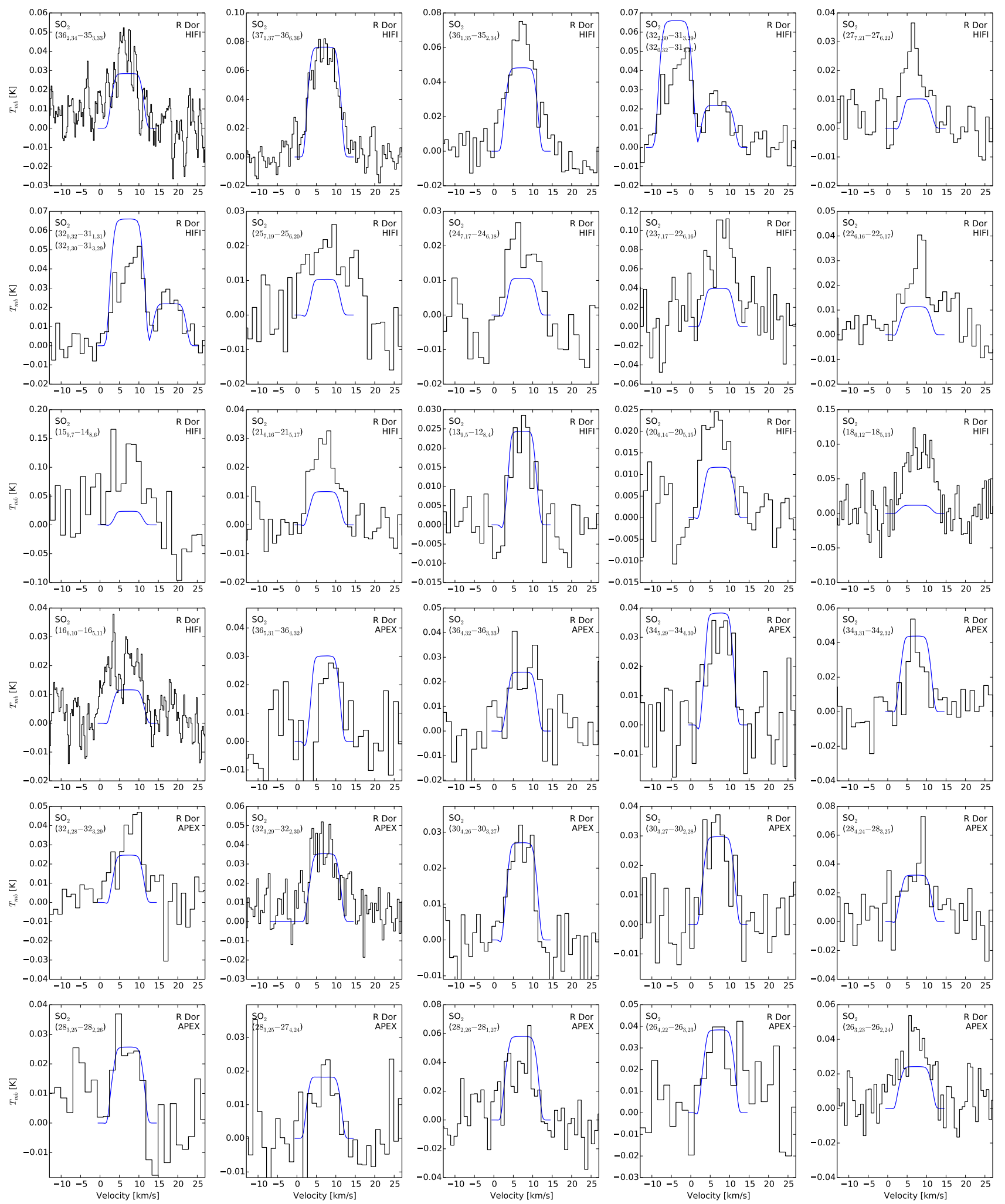

Fig. A.1. $\mathrm{SO}_{2}$ model (blue line) and observations (black histograms) of $\mathrm{R}$ Dor. In the case of overlapping lines, the top line listed is always the line centred at $v_{\mathrm{LSR}}=5.7 \mathrm{~km} \mathrm{~s}^{-1}$. 
T. Danilovich et al.: Sulphur molecules in the circumstellar envelopes of M-type AGB stars
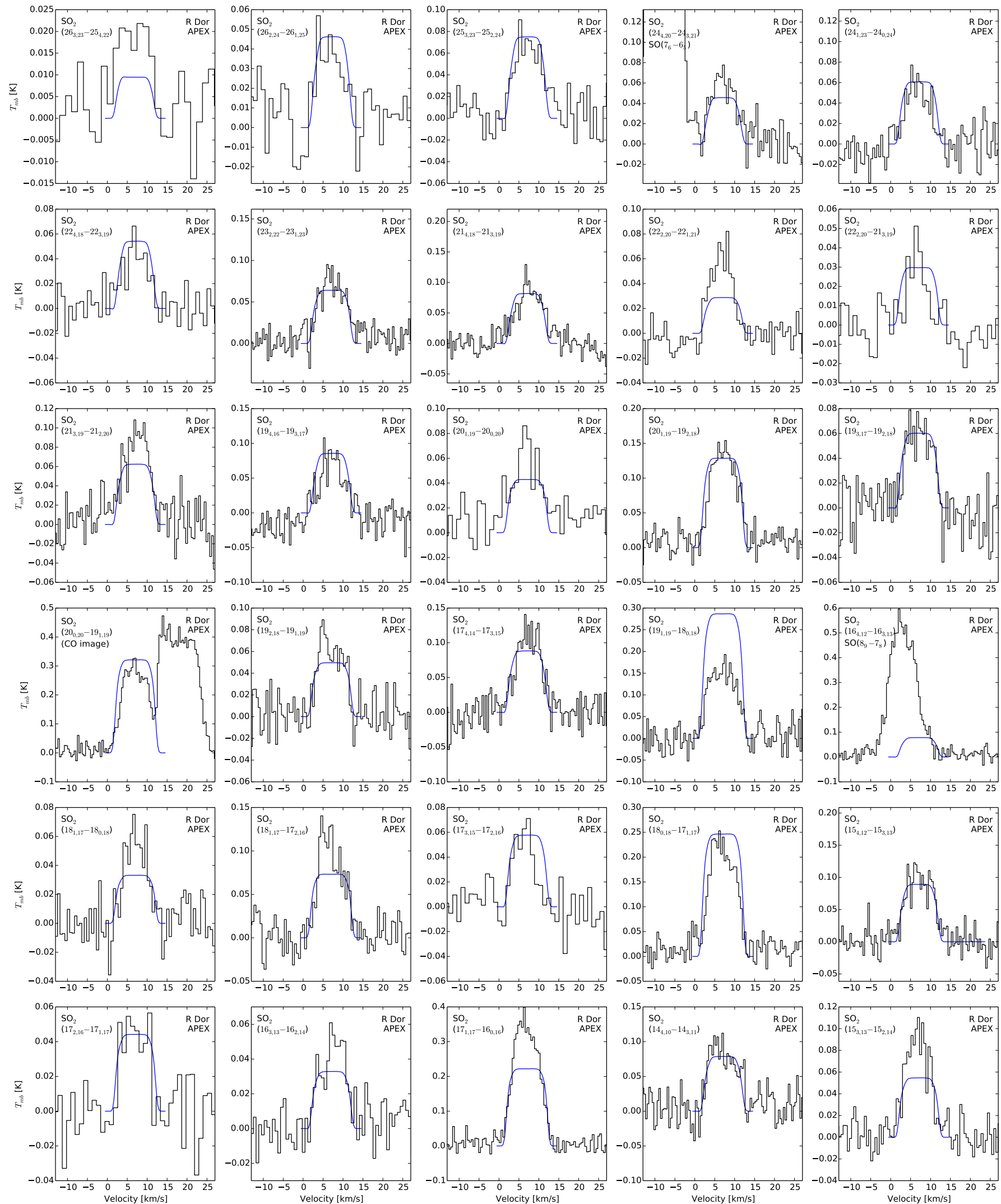

Fig. A.1. continued. 

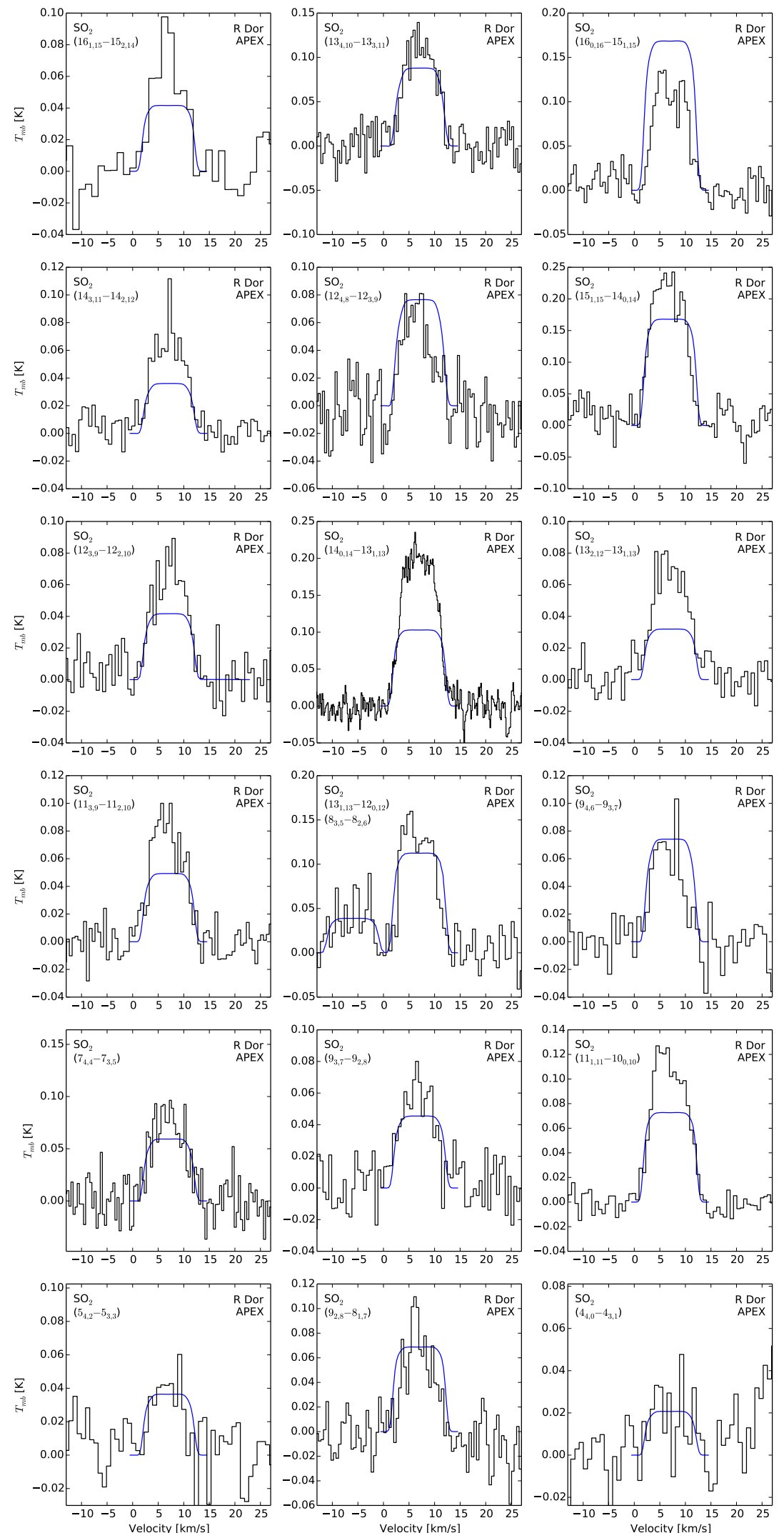

$=0.15$

$0.000 \ln 2 \ln \mid]^{2}$

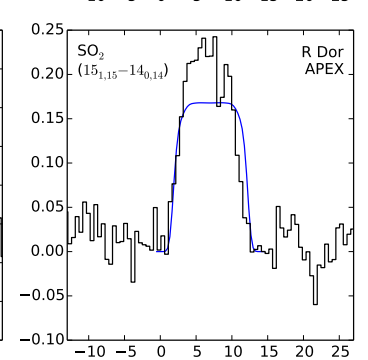
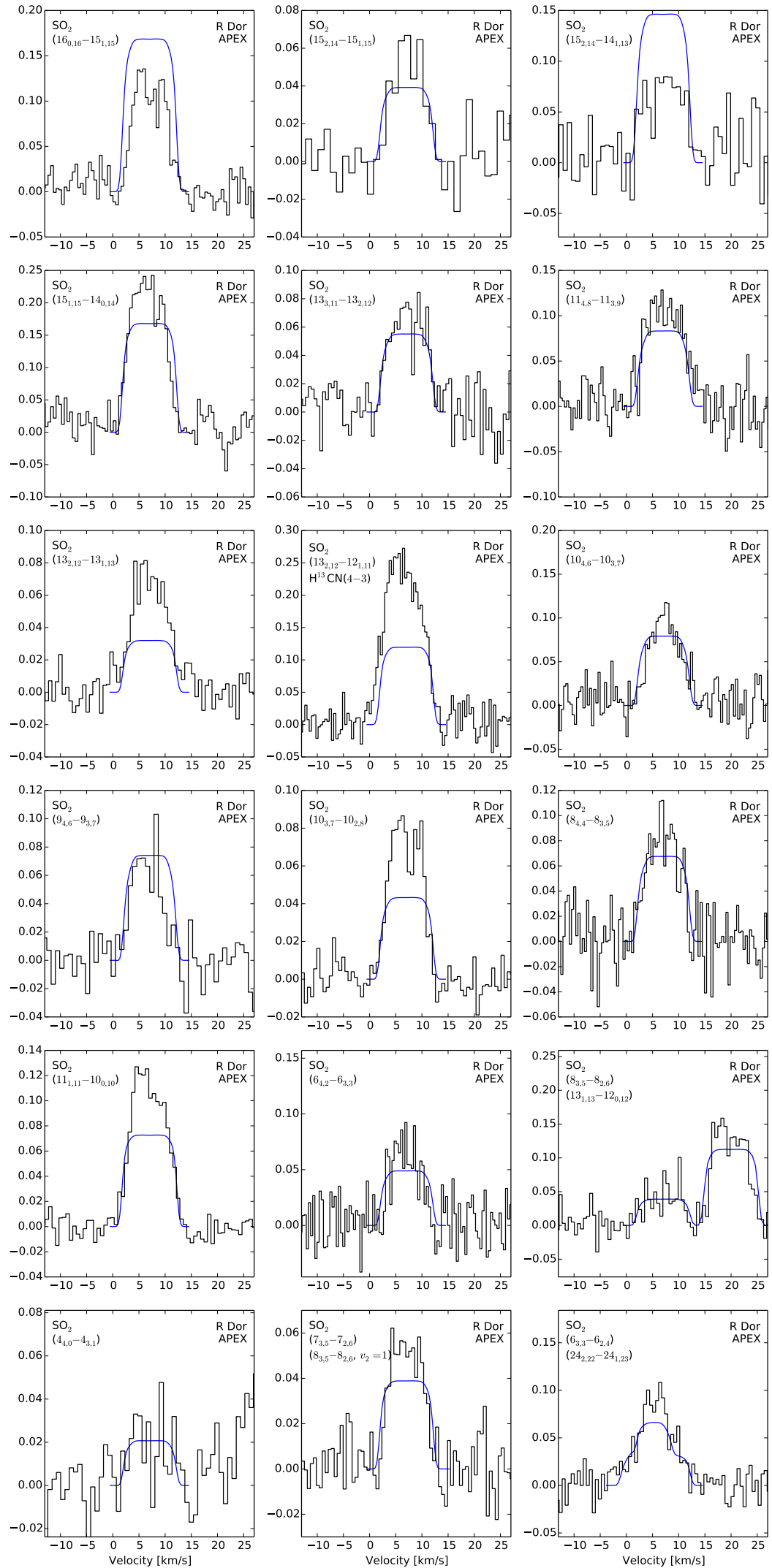

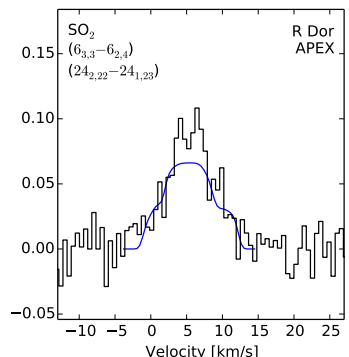

Fig. A.1. continued. 
T. Danilovich et al.: Sulphur molecules in the circumstellar envelopes of M-type AGB stars
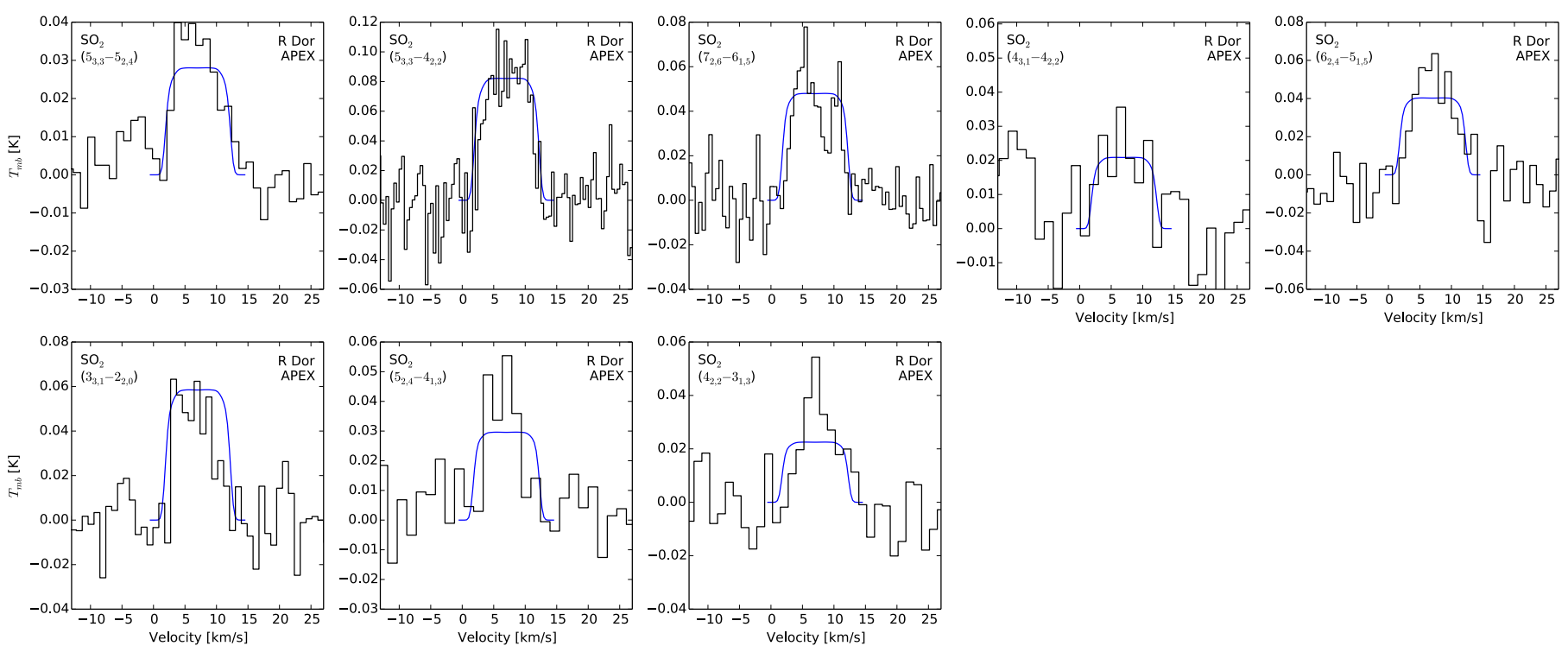

Fig. A.1. continued. 
A\&A 588, A119 (2016)
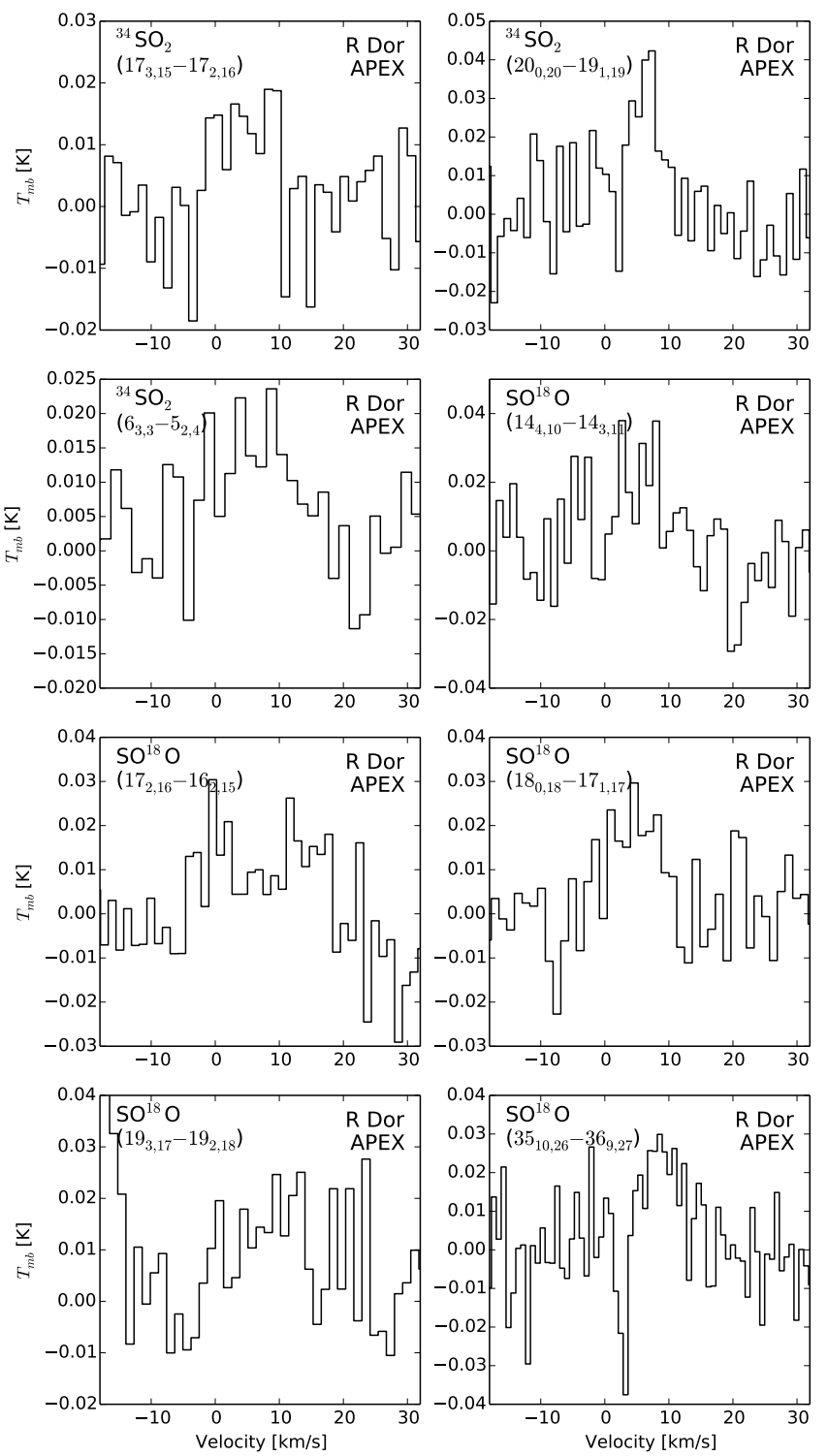

Fig. A.2. Detections of the isotopologues ${ }^{34} \mathrm{SO}_{2}$ and $\mathrm{SO}^{18} \mathrm{O}$ towards R Dor.

\section{Appendix B: HIFI OBSIDs}

The observation IDs for the HIFI observations used in this work are given in Table B.1, including the non-detections used to constrain our TX Cam SO model.

Table B.1. HIFI OBSIDs for observations used in this work.

\begin{tabular}{cc}
\hline \hline Star & OBSID \\
\hline IK Tau & 1342190198 \\
& 1342191594 \\
R Dor & 1342198355 \\
& 1342197982 \\
& 1342200969 \\
TX Cam & 1342200906 \\
& $1342205330^{*}$ \\
W Hya & $1342205309^{*}$ \\
& 1342200951 \\
R Cas & 1342200981 \\
& 1342200929 \\
& 1342198335 \\
\hline
\end{tabular}

Notes. ${ }^{(*)}$ Indicates only upper limits were derived for the SO lines. 
T. Danilovich et al.: Sulphur molecules in the circumstellar envelopes of M-type AGB stars

\section{Appendix C: Additional tables}

Table C.1. $\mathrm{SO}_{2}$ observations towards R Dor using APEX, listed in order of descending energy of the upper level.

\begin{tabular}{|c|c|c|c|c|c|c|c|c|c|}
\hline Transition & $\begin{array}{c}v \\
{[\mathrm{GHz}]}\end{array}$ & $\begin{array}{l}E_{\text {up }} \\
{[\mathrm{K}]}\end{array}$ & $\begin{array}{c}\theta \\
{\left[{ }^{\prime \prime}\right]}\end{array}$ & $\begin{array}{c}I_{\mathrm{mb}} \\
{\left[\mathrm{K} \mathrm{km} \mathrm{s}^{-1}\right]}\end{array}$ & Transition & $\begin{array}{c}v \\
{[\mathrm{GHz}]}\end{array}$ & $\begin{array}{l}E_{\text {up }} \\
{[\mathrm{K}]}\end{array}$ & $\begin{array}{c}\theta \\
{\left[{ }^{\prime \prime}\right]}\end{array}$ & $\begin{array}{c}I_{\mathrm{mb}} \\
{\left[\mathrm{K} \mathrm{km} \mathrm{s}^{-1}\right]}\end{array}$ \\
\hline $25_{4,22} \rightarrow 26_{1,25}$ & * 279.497 & 1085 & 22 & 0.373 & $16_{3,13} \rightarrow 16_{2,14}$ & 214.689 & 148 & 29 & 0.415 \\
\hline $40_{4,36} \rightarrow 40_{3,37}$ & 341.403 & 808 & 18 & 0.217 & $17_{1,17} \rightarrow 16_{0,16}$ & 313.661 & 136 & 20 & 2.881 \\
\hline $36_{5,31} \rightarrow 36_{4,32}$ & 341.674 & 679 & 18 & 0.148 & $14_{4,10} \rightarrow 14_{3,11}$ & 351.874 & 136 & 18 & 0.860 \\
\hline $36_{4,32} \rightarrow 36_{3,33}$ & 281.689 & 662 & 22 & 0.257 & $15_{3,13} \rightarrow 15_{2,14}$ & 275.240 & 133 & 23 & 0.745 \\
\hline $34_{5,29} \rightarrow 34_{4,30}$ & 360.290 & 612 & 17 & 0.250 & $16_{1,15} \rightarrow 15_{2,14}$ & 236.217 & 131 & 26 & 0.595 \\
\hline $34_{3,31} \rightarrow 34_{2,32}$ & 342.762 & 582 & 18 & 0.255 & $13_{4,10} \rightarrow 13_{3,11}$ & 357.165 & 123 & 17 & 0.919 \\
\hline $32_{4,28} \rightarrow 32_{3,29}$ & 258.389 & 531 & 24 & 0.368 & $16_{0,16} \rightarrow 15_{1,15}$ & 283.292 & 121 & 22 & 0.959 \\
\hline $32_{3,29} \rightarrow 32_{2,30}$ & 300.273 & 519 & 21 & 0.453 & $15_{2,14} \rightarrow 15_{1,15}$ & 248.057 & 119 & 25 & 0.423 \\
\hline $30_{4,26} \rightarrow 30_{3,27}$ & 259.599 & 472 & 24 & 0.212 & $15_{2,14} \rightarrow 14_{1,13}$ & 366.214 & 119 & 17 & 0.840 \\
\hline $30_{3,27} \rightarrow 30_{2,28}$ & 263.544 & 459 & 24 & 0.231 & $14_{3,11} \rightarrow 14_{2,12}$ & 226.300 & 119 & 28 & 0.596 \\
\hline $28_{4,24} \rightarrow 28_{3,25}$ & 267.720 & 416 & 23 & 0.412 & $12_{4,8} \rightarrow 12_{3,9}$ & 355.046 & 111 & 18 & 0.498 \\
\hline $28_{3,25} \rightarrow 28_{2,26}$ & 234.187 & 403 & 27 & 0.289 & $13_{3,11} \rightarrow 13_{2,12}$ & 267.537 & 106 & 23 & 0.600 \\
\hline $28_{3,25} \rightarrow 27_{4,24}$ & 313.412 & 403 & 20 & 0.129 & $15_{1,15} \rightarrow 14_{0,14}$ & 281.763 & 107 & 22 & 2.007 \\
\hline $28_{2,26} \rightarrow 28_{1,27}$ & 340.316 & 392 & 18 & 0.365 & $11_{4,8} \rightarrow 11_{3,9}$ & 357.388 & 100 & 17 & 0.980 \\
\hline $26_{4,22} \rightarrow 26_{3,23}$ & 280.807 & 364 & 22 & 0.382 & $12_{3,9} \rightarrow 12_{2,10}$ & 237.069 & 94 & 26 & 0.616 \\
\hline $26_{3,23} \rightarrow 26_{2,24}$ & 213.068 & 351 & 29 & 0.410 & $14_{0,14} \rightarrow 13_{1,13}$ & 244.254 & 94 & 26 & 1.702 \\
\hline $26_{3,23} \rightarrow 25_{4,22}$ & 245.339 & 351 & 25 & 0.216 & $13_{2,12} \rightarrow 13_{1,13}$ & 225.154 & 93 & 28 & 0.652 \\
\hline $26_{2,24} \rightarrow 26_{1,25}$ & 296.169 & 341 & 21 & 0.306 & $13_{2,12} \rightarrow 12_{1,11}$ & $\dagger 345.339$ & 93 & 18 & 2.283 \\
\hline $25_{3,23} \rightarrow 25_{2,24}$ & 359.151 & 321 & 17 & 0.601 & $10_{4,6} \rightarrow 10_{3,7}$ & 356.755 & 90 & 17 & 0.804 \\
\hline $24_{4,20} \rightarrow 24_{3,21}$ & $\dagger 296.535$ & 317 & 21 & 0.520 & $11_{3,9} \rightarrow 11_{2,10}$ & 262.257 & 83 & 24 & 0.639 \\
\hline $24_{1,23} \rightarrow 24_{0,24}$ & 363.891 & 281 & 17 & 0.396 & $13_{1,13} \rightarrow 12_{0,12}$ & $\dagger 251.200$ & 82 & 25 & 1.275 \\
\hline $22_{4,18} \rightarrow 22_{3,19}$ & 312.543 & 273 & 20 & 0.424 & $9_{4,6} \rightarrow 9_{3,7}$ & 357.672 & 81 & 17 & 0.497 \\
\hline $23_{2,22} \rightarrow 23_{1,23}$ & 363.926 & 260 & 17 & 0.741 & $10_{3,7} \rightarrow 10_{2,8}$ & 245.563 & 73 & 25 & 0.587 \\
\hline $21_{4,18} \rightarrow 21_{3,19}$ & 363.159 & 252 & 17 & 0.815 & $8_{4,4} \rightarrow 8_{3,5}$ & 357.581 & 72 & 17 & 0.686 \\
\hline $22_{2,20} \rightarrow 22_{1,21}$ & 216.643 & 248 & 29 & 0.373 & $7_{4,4} \rightarrow 7_{3,5}$ & 357.892 & 65 & 17 & 0.685 \\
\hline $22_{2,20} \rightarrow 21_{3,19}$ & 286.416 & 248 & 22 & 0.168 & $9_{3,7} \rightarrow 9_{2,8}$ & 258.942 & 64 & 24 & 0.561 \\
\hline $21_{3,19} \rightarrow 21_{2,20}$ & 316.099 & 235 & 20 & 0.878 & $11_{1,11} \rightarrow 10_{0,10}$ & 221.965 & 60 & 28 & 0.953 \\
\hline $19_{4,16} \rightarrow 19_{3,17}$ & 359.771 & 214 & 17 & 0.430 & $6_{4,2} \rightarrow 6_{3,3}$ & 357.926 & 59 & 17 & 0.527 \\
\hline $20_{1,19} \rightarrow 20_{0,20}$ & 282.293 & 199 & 22 & 0.751 & $8_{3,5} \rightarrow 8_{2,6}$ & $\dagger 251.211$ & 55 & 25 & 0.995 \\
\hline $20_{1,19} \rightarrow 19_{2,18}$ & 338.612 & 199 & 18 & 1.255 & $5_{4,2} \rightarrow 5_{3,3}$ & 358.013 & 53 & 17 & 0.398 \\
\hline $19_{3,17} \rightarrow 19_{2,18}$ & 299.317 & 197 & 21 & 0.496 & $9_{2,8} \rightarrow 8_{1,7}$ & 298.576 & 51 & 21 & 0.496 \\
\hline $20_{0,20} \rightarrow 19_{1,19}$ & $\dagger 358.216$ & 185 & 17 & 2.373 & $4_{4,0} \rightarrow 4_{3,1}$ & 358.038 & 49 & 17 & 0.234 \\
\hline $19_{2,18} \rightarrow 19_{1,19}$ & 301.897 & 183 & 21 & 0.561 & $7_{3,5} \rightarrow 7_{2,6}$ & 257.100 & 48 & 24 & 0.448 \\
\hline $17_{4,14} \rightarrow 17_{3,15}$ & 357.963 & 180 & 17 & 0.917 & $6_{3,3} \rightarrow 6_{2,4}$ & $\dagger 254.281$ & 41 & 25 & 0.776 \\
\hline $19_{1,19} \rightarrow 18_{0,18}$ & 346.652 & 168 & 18 & 1.390 & $5_{3,3} \rightarrow 5_{2,4}$ & 256.247 & 36 & 24 & 0.350 \\
\hline $16_{4,12} \rightarrow 16_{3,13}$ & 346.524 & 165 & 18 & 3.864 & $5_{3,3} \rightarrow 4_{2,2}$ & 351.257 & 36 & 18 & 0.705 \\
\hline $18_{1,17} \rightarrow 18_{0,18}$ & 240.943 & 163 & 26 & 0.506 & $7_{2,6} \rightarrow 6_{1,5}$ & 271.529 & 36 & 23 & 0.426 \\
\hline $18_{1,17} \rightarrow 17_{2,16}$ & 288.520 & 163 & 22 & 0.852 & $4_{3,1} \rightarrow 4_{2,2}$ & 255.553 & 31 & 24 & 0.215 \\
\hline $17_{3,15} \rightarrow 17_{2,16}$ & 285.744 & 163 & 22 & 0.382 & $6_{2,4} \rightarrow 5_{1,5}$ & 282.037 & 29 & 22 & 0.349 \\
\hline $18_{0,18} \rightarrow 17_{1,17}$ & 321.330 & 152 & 19 & 2.091 & $3_{3,1} \rightarrow 2_{2,0}$ & 313.280 & 28 & 20 & 0.381 \\
\hline $15_{4,12} \rightarrow 15_{3,13}$ & 357.241 & 150 & 17 & 0.893 & $5_{2,4} \rightarrow 4_{1,3}$ & 241.616 & 24 & 26 & 0.398 \\
\hline $17_{2,16} \rightarrow 17_{1,17}$ & 273.753 & 149 & 23 & 0.378 & $4_{2,2} \rightarrow 3_{1,3}$ & 235.152 & 19 & 27 & 0.211 \\
\hline
\end{tabular}

Notes. ${ }^{(*)}$ Indicates a $v_{2}=1$ transition; ${ }^{(\dagger)}$ indicates a line overlap and hence an approximate line intensity. 
A\&A 588, A119 (2016)

Table C.2. $\mathrm{SO}_{2}$ and $\mathrm{SO}$ observations using HIFI, listed by molecule in order of descending energy of the upper level.

\begin{tabular}{|c|c|c|c|c|c|c|c|c|c|}
\hline Molecule & Transition & $\begin{array}{c}v \\
{[\mathrm{GHz}]}\end{array}$ & $\begin{array}{l}E_{\text {up }} \\
{[\mathrm{K}]}\end{array}$ & $\begin{array}{c}\theta \\
{\left[{ }^{\prime \prime}\right]}\end{array}$ & $\begin{array}{c}\text { IK Tau } \\
{\left[\mathrm{K} \mathrm{km} \mathrm{s}^{-1}\right]}\end{array}$ & $\begin{array}{c}\text { R Dor } \\
{\left[\mathrm{K} \mathrm{km} \mathrm{s}^{-1}\right]}\end{array}$ & $\begin{array}{c}\text { TX Cam } \\
{\left[\mathrm{K} \mathrm{km} \mathrm{s}^{-1}\right]}\end{array}$ & $\begin{array}{c}\text { W Hya } \\
{\left[\mathrm{K} \mathrm{km} \mathrm{s}^{-1}\right]}\end{array}$ & $\begin{array}{c}\mathrm{R} \mathrm{Cas} \\
{\left[\mathrm{K} \mathrm{km} \mathrm{s}^{-1}\right]}\end{array}$ \\
\hline \multirow[t]{5}{*}{ SO } & $23_{24} \rightarrow 22_{23}$ & 988.618 & 575 & 21 & - & - & - & 0.76 & - \\
\hline & $15_{16} \rightarrow 14_{15}$ & 645.875 & 253 & 33 & - & 1.5 & $<0.1$ & 0.43 & 0.13 \\
\hline & $13_{13} \rightarrow 12_{12}$ & 559.319 & 201 & 37 & 0.16 & 1.3 & $<0.08$ & 0.55 & 0.16 \\
\hline & $13_{12} \rightarrow 12_{11}$ & 558.087 & 195 & 37 & 0.44 & 1.4 & $<0.08$ & 0.42 & 0.14 \\
\hline & $13_{14} \rightarrow 12_{13}$ & 560.178 & 193 & 37 & 0.23 & 1.5 & $<0.08$ & 0.58 & 0.26 \\
\hline \multirow[t]{16}{*}{$\mathrm{SO}_{2}$} & $36_{2,34} \rightarrow 35_{3,33}$ & $* 661.510$ & 630 & 32 & - & 0.37 & - & - & - \\
\hline & $37_{1,37} \rightarrow 36_{0,36}$ & 659.421 & 609 & 32 & - & 0.68 & - & 0.12 & - \\
\hline & $36_{1,35} \rightarrow 35_{2,34}$ & 658.632 & 606 & 32 & 0.16 & 0.61 & - & 0.35 & 0.26 \\
\hline & $32_{2,30} \rightarrow 31_{3,29}$ & 571.532 & 505 & 36 & - & $0.20^{\dagger}$ & - & - & - \\
\hline & $27_{7,21} \rightarrow 27_{6,22}$ & $* 657.885$ & 468 & 32 & - & 0.21 & - & - & - \\
\hline & $32_{0,32} \rightarrow 31_{1,31}$ & 571.553 & 459 & 36 & - & $0.39^{\dagger}$ & - & - & - \\
\hline & $25_{7,19} \rightarrow 25_{6,20}$ & $* 659.338$ & 419 & 32 & - & 0.25 & - & - & - \\
\hline & $24_{7,17} \rightarrow 24_{6,18}$ & 659.898 & 396 & 32 & - & $0.23^{\dagger}$ & - & - & - \\
\hline & $23_{7,17} \rightarrow 22_{6,16}$ & 1102.115 & 374 & 19 & - & 0.93 & - & - & - \\
\hline & $22_{6,16} \rightarrow 22_{5,17}$ & 557.283 & 321 & 37 & - & 0.29 & - & 0.14 & - \\
\hline & $15_{9,7} \rightarrow 14_{8,6}$ & 1151.852 & 309 & 19 & - & 1.5 & - & - & - \\
\hline & $21_{6,16} \rightarrow 21_{5,17}$ & 558.391 & 301 & 37 & - & 0.22 & - & 0.20 & - \\
\hline & $13_{9,5} \rightarrow 12_{8,4}$ & 1113.506 & 282 & 19 & - & 0.76 & - & - & - \\
\hline & $20_{6,14} \rightarrow 20_{5,15}$ & 558.812 & 282 & 37 & - & 0.14 & - & - & - \\
\hline & $18_{6,12} \rightarrow 18_{5,13}$ & 559.882 & 246 & 37 & - & 0.19 & - & 0.12 & - \\
\hline & $16_{6,10} \rightarrow 16_{5,11}$ & 560.613 & 213 & 37 & - & 0.22 & - & - & - \\
\hline
\end{tabular}

Notes. ${ }^{(*)}$ Indicates a line not listed in Justtanont et al. $(2012) ;{ }^{(\dagger)}$ indicates a line blend and hence an approximate integrated intensity.

Table C.3. Archival observations of $\mathrm{SO}$ and $\mathrm{SO}_{2}$ towards IK Tau, TX Cam, R Cas, and W Hya.

\begin{tabular}{|c|c|c|c|c|c|c|c|c|}
\hline Source & Molecule & Transition & $\begin{array}{c}v \\
{[\mathrm{GHz}]}\end{array}$ & $\begin{array}{l}E_{\mathrm{up}} \\
{[\mathrm{K}]}\end{array}$ & Telescope & $\begin{array}{c}\theta \\
{\left[{ }^{\prime \prime}\right]}\end{array}$ & $\begin{array}{c}I_{\mathrm{mb}} \\
{\left[\mathrm{K} \mathrm{km} \mathrm{s}^{-1}\right]}\end{array}$ & Reference \\
\hline \multirow{20}{*}{ IK Tau } & \multirow{7}{*}{ SO } & $8_{8} \rightarrow 77$ & 344.310 & 88 & APEX & 18 & 2.7 & Kim et al. (2010) \\
\hline & & $7_{7} \rightarrow 6_{6}$ & 301.286 & 71 & APEX & 20 & 0.89 & Kim et al. (2010) \\
\hline & & $5_{6} \rightarrow 4_{5}$ & 219.949 & 35 & NRAO & 30 & 6.5 & Sahai \& Wannier (1992) \\
\hline & & $2_{2} \rightarrow 1_{1}$ & 86.094 & 19 & IRAM & 27 & 0.65 & Omont et al. (1993) \\
\hline & & $3_{4} \rightarrow 2_{3}$ & 138.179 & 16 & IRAM & 17 & 13.5 & Sahai \& Wannier (1992) \\
\hline & & $2_{3} \rightarrow 1_{2}$ & 99.300 & 9 & OSO & 37.5 & 4.2 & Sahai \& Wannier (1992) \\
\hline & & $2_{3} \rightarrow 1_{2}$ & 99.300 & 9 & OSO & 37.5 & 3.6 & Olofsson et al. (1998) \\
\hline & \multirow[t]{13}{*}{$\mathrm{SO}_{2}$} & $17_{1,17} \rightarrow 16_{0,16}$ & 313.660 & 136 & APEX & 20 & 11.3 & Kim et al. (2010) \\
\hline & & $14_{4,10} \rightarrow 14_{3,11}$ & 351.873 & 136 & APEX & 18 & 0.55 & Kim et al. (2010) \\
\hline & & $14_{3,11} \rightarrow 14_{2,12}$ & 226.300 & 119 & IRAM & 10.5 & 1.0 & Decin et al. (2010a) \\
\hline & & $13_{2,12} \rightarrow 12_{1,11}$ & 345.338 & 93 & APEX & 18 & 6.3 & Kim et al. (2010) \\
\hline & & $10_{1,9} \rightarrow 10_{0,10}$ & 104.239 & 55 & IRAM & 24 & 1.83 & Omont et al. (1993) \\
\hline & & $10_{0,10} \rightarrow 9_{1,9}$ & 160.828 & 50 & IRAM & 15 & 8.6 & Omont et al. (1993) \\
\hline & & $5_{3,3} \rightarrow 5_{2,4}$ & 256.247 & 36 & IRAM & 9.5 & 3.4 & Decin et al. (2010a) \\
\hline & & $5_{3,3} \rightarrow 4_{2,2}$ & 351.257 & 36 & APEX & 18 & 1.4 & Kim et al. (2010) \\
\hline & & $4_{3,1} \rightarrow 4_{2,2}$ & 255.553 & 31 & IRAM & 9.5 & 3.2 & Decin et al. (2010a) \\
\hline & & $4_{3,1} \rightarrow 3_{2,2}$ & 332.505 & 31 & APEX & 19 & 1.4 & Kim et al. (2010) \\
\hline & & $3_{3,1} \rightarrow 3_{2,2}^{2,2}$ & 255.958 & 28 & IRAM & 9.5 & 2.2 & Decin et al. (2010a) \\
\hline & & $3_{3,1} \rightarrow 2,2$ & 313.279 & 28 & APEX & 20 & 2.2 & Kim et al. (2010) \\
\hline & & $3_{1,3} \rightarrow 2_{0,2}$ & 104.029 & 8 & IRAM & 24 & 1.78 & Omont et al. (1993) \\
\hline \multirow[t]{4}{*}{ TX Cam } & \multirow[t]{4}{*}{$\mathrm{SO}$} & $5_{6} \rightarrow 4_{5}$ & 219.949 & 35 & IRAM & 13 & 7.0 & Bujarrabal et al. (1994) \\
\hline & & $5_{6} \rightarrow 4_{5}$ & 219.949 & 35 & NRAO & 30 & 1.8 & Sahai \& Wannier (1992) \\
\hline & & $2_{3} \rightarrow 1_{2}$ & 99.300 & 9 & OSO & 37.5 & 2.9 & Sahai \& Wannier (1992) \\
\hline & & $2_{3} \rightarrow 1_{2}$ & 99.300 & 9 & OSO & 37.5 & 2.1 & Olofsson et al. (1998) \\
\hline \multirow[t]{2}{*}{ W Hya } & \multirow[t]{2}{*}{$\mathrm{SO}$} & $5_{5} \rightarrow 4_{4}$ & 215.221 & 31 & SMA & 1.5 & $8.2^{*}$ & Vlemmings et al. (2011) \\
\hline & & $2_{3} \rightarrow 1_{2}$ & 99.300 & 9 & SEST & 51 & 0.2 & Olofsson et al. (1998) \\
\hline \multirow[t]{4}{*}{ R Cas } & \multirow[t]{3}{*}{$\mathrm{SO}$} & $5_{6} \rightarrow 4_{5}$ & 219.949 & 35 & NRAO & 30 & 2.0 & Sahai \& Wannier (1992) \\
\hline & & $2_{3} \rightarrow 1_{2}$ & 99.300 & 9 & OSO & 37.5 & 1.4 & Sahai \& Wannier (1992) \\
\hline & & $2_{3} \rightarrow 1_{2}$ & 99.300 & 9 & OSO & 37.5 & 1.7 & Olofsson et al. (1998) \\
\hline & $\mathrm{SO}_{2}$ & $3_{1,3} \rightarrow 2_{0,2}$ & 104.029 & 7.7 & IRAM & 24 & 0.81 & Guilloteau et al. (1986) \\
\hline
\end{tabular}

Notes. ${ }^{(*)}$ Indicates value given is the flux in $\mathrm{Jy}_{\mathrm{km} \mathrm{s}}{ }^{-1}$ not the main beam integrated intensity. 